University of Tennessee Health Science Center UTHSC Digital Commons

\title{
$5-2013$
}

\section{Effects of Noise on Cognitive Function During Dual Tasks across Normally Aging Adults}

Jennine Harvey

University of Tennessee Health Science Center

Follow this and additional works at: https://dc.uthsc.edu/dissertations

Part of the Speech and Hearing Science Commons, and the Speech Pathology and Audiology Commons

\section{Recommended Citation}

Harvey, Jennine, "Effects of Noise on Cognitive Function During Dual Tasks across Normally Aging Adults" (2013). Theses and Dissertations (ETD). Paper 106. http://dx.doi.org/10.21007/ etd.cghs.2013.0129.

This Dissertation is brought to you for free and open access by the College of Graduate Health Sciences at UTHSC Digital Commons. It has been accepted for inclusion in Theses and Dissertations (ETD) by an authorized administrator of UTHSC Digital Commons. For more information, please contact jwelch30@uthsc.edu. 


\title{
Effects of Noise on Cognitive Function During Dual Tasks across Normally Aging Adults
}

\author{
Abstract \\ This study expands upon methods used to investigate cognition and speech perception which have been \\ limited by lack of (a) pre-screening of cognitive function in participants, (b) reporting visual or auditory \\ accuracy and reaction time measures across experimental conditions that examine reaction times, and \\ (c) comprehensive test battery that includes performance across multiple levels of dual task paradigms \\ (DTPs). This study aims to examine group performance on dual tasks (DT) increasing in cognitive (task \\ difficulty) and perceptual load (noise) with age. Participants were divided into two groups based upon \\ age. Group 1 consisted of 14 listeners (Female $=11$ ) who were 40-59 years old (Mean=53.18, SD=5.97). \\ Group 2 consisted of 15 listeners (Female=9) who were 60 years old and older (Mean=72.07, SD=5.11). \\ All participants were tested in each of 3 experimental conditions: (1) auditory word recognition + visual \\ processing, (2) auditory working memory (word) + visual processing, and (3) auditory working memory \\ (sentence) + visual processing in noise. The results indicate auditory accuracy, visual accuracy, and visual \\ processing speed are affected by the complexity of the given task.

\section{Document Type} \\ Dissertation

\section{Degree Name} \\ Doctor of Philosophy (PhD)

\section{Program} \\ Audiology and Speech-Language Pathology

\section{Research Advisor} \\ Deborah von Hapsburg, Ph.D.

\section{Keywords} \\ Cognition, Dual Task, Elderly, Noise

\section{Subject Categories} \\ Communication Sciences and Disorders | Medicine and Health Sciences | Speech and Hearing Science | \\ Speech Pathology and Audiology
}




\title{
Effects of Noise on Cognitive Function during Dual Tasks across Normally Aging Adults
}

\author{
A Dissertation \\ Presented for \\ The Graduate Studies Council \\ The University of Tennessee \\ Health Science Center
}

In Partial Fulfillment

Of the Requirements for the Degree

Doctor of Philosophy

From The University of Tennessee

By

Jennine Harvey

May 2013 
Copyright (C) 2013 by Jennine Harvey. All rights reserved. 


\section{DEDICATION}

I would like to dedicate this work to my family and friends. First and foremost, to my mother, Barbara Harvey, your unwavering love and support brought me to this moment-Mom, this one is for you. To my amazing siblings, Tim, Katie, and Ashley: you always manage to keep life interesting and filled with laughter.

I also dedicate this to my dear friends who have supported me through this process. I can never repay you for all you have given. I would like to give special thanks to Tia Nicole Friedman, who has been my best friend and confidante for the last eighteen years; Merideth Kongchan, who has been my safe house in the storm; and Megan Cuellar, whose tenacity, strength, and fearlessness inspire me every day. M., thank you for being my partner in crime.

Lastly, I dedicate this to my incredible husband, D. Ryan Northrop, whose strength, love, and support made this day possible. Thank you for everyday, for being my light in the darkness. 


\section{ACKNOWLEDGEMENTS}

I would like to thank my committee members who were so generous of their time and expertise. A special thanks to Dr. Deborah von Hapsburg, my committee chair, for her countless hours of reading, Skyping, and encouraging. Most of all, I would like to thank her for her patience throughout the entire process. Thank you Dr. Tim Saltuklaroglu, Dr. Kristin King, Dr. Patrick Plyler, and Dr. Gregory Petty for your incredible support and agreeing to serve on my committee.

I would like to acknowledge and thank The University of Tennessee-Knoxville for allowing me to conduct my research and providing any assistance requested. Special thanks go to the Knoxville, Tennessee, community and senior centers, including the John O'Connor Senior Center and the Halls Senior Center, for their continued support. 


\begin{abstract}
This study expands upon methods used to investigate cognition and speech perception which have been limited by lack of (a) pre-screening of cognitive function in participants, (b) reporting visual or auditory accuracy and reaction time measures across experimental conditions that examine reaction times, and (c) comprehensive test battery that includes performance across multiple levels of dual task paradigms (DTPs). This study aims to examine group performance on dual tasks (DT) increasing in cognitive (task difficulty) and perceptual load (noise) with age. Participants were divided into two groups based upon age. Group 1 consisted of 14 listeners (Female=11) who were 40-59 years old (Mean=53.18, SD=5.97). Group 2 consisted of 15 listeners (Female=9) who were 60 years old and older (Mean=72.07, $\mathrm{SD}=5.11)$. All participants were tested in each of 3 experimental conditions: (1) auditory word recognition + visual processing, (2) auditory working memory (word) + visual processing, and (3) auditory working memory (sentence) + visual processing in noise. The results indicate auditory accuracy, visual accuracy, and visual processing speed are affected by the complexity of the given task.
\end{abstract}




\section{TABLE OF CONTENTS}

CHAPTER 1. INTRODUCTION .....................................................................................1

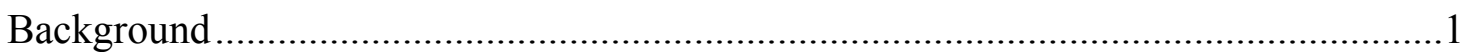

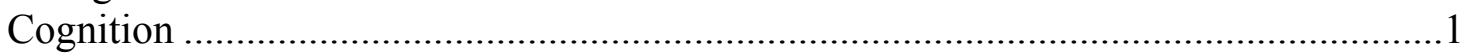

The Effect of Age on Cognition and the Brain ............................................................2

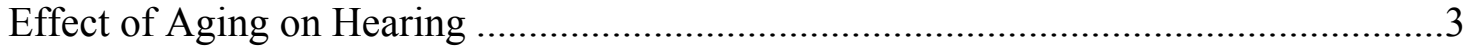

Perception and Cognitive Load Model ....................................................................4

CHAPTER 2. LITERATURE REVIEW .................................................................9

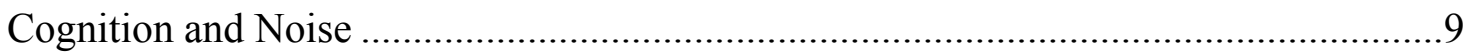

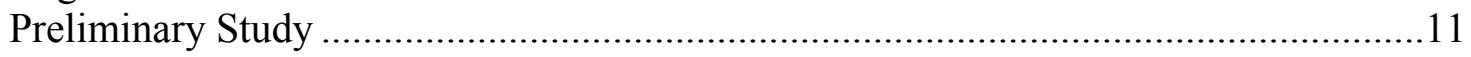

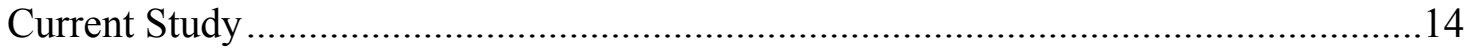

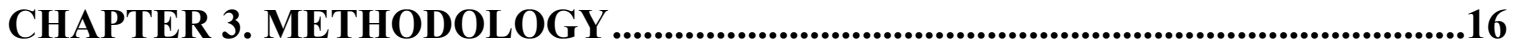

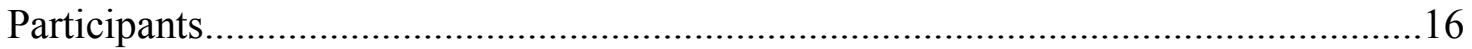

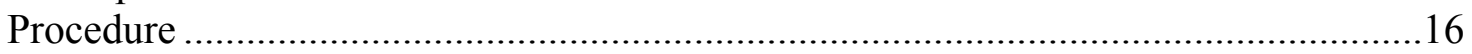

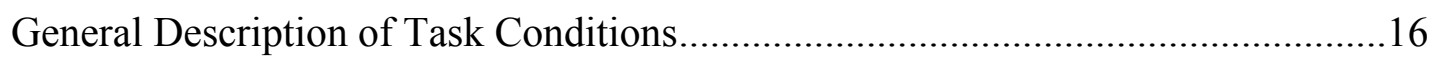

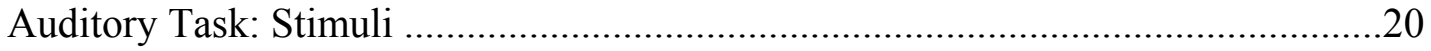

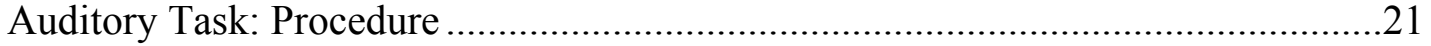

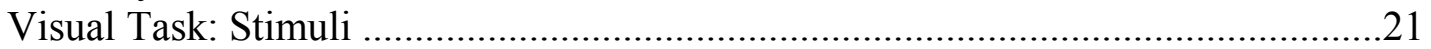

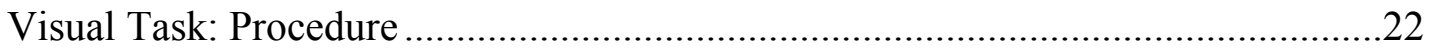

Simple Dual Task Condition.............................................................................22

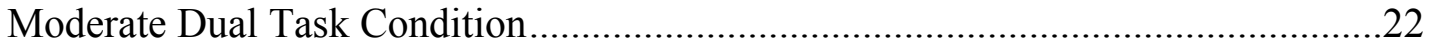

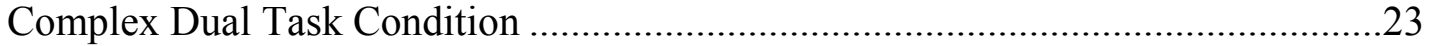

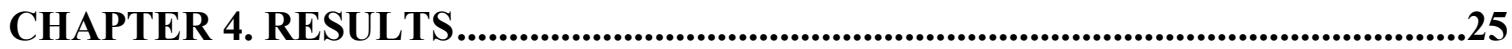

Doubly Repeated Mixed Multivariate Analysis ..................................................25

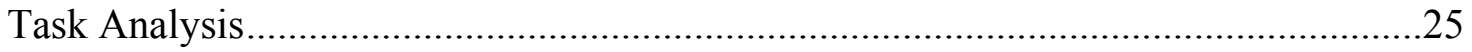

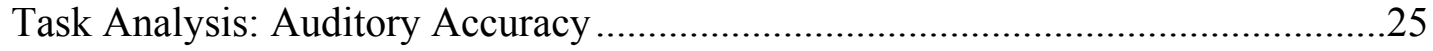

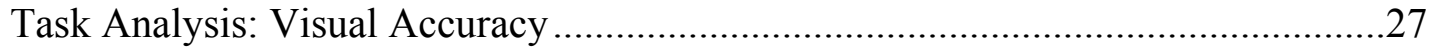

Task Analysis: Reaction Time .....................................................................27

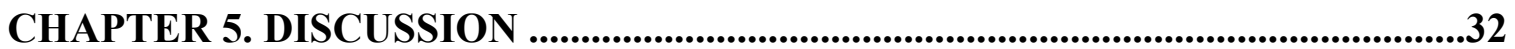

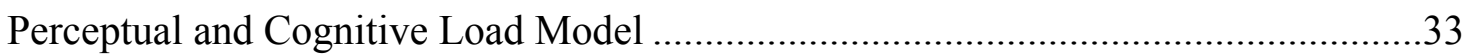

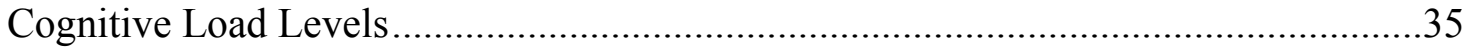

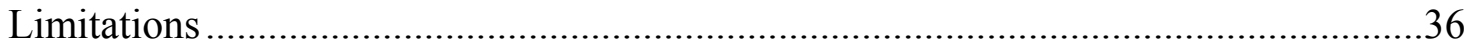

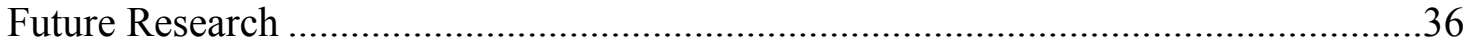

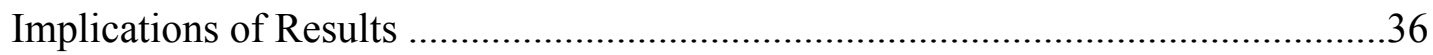

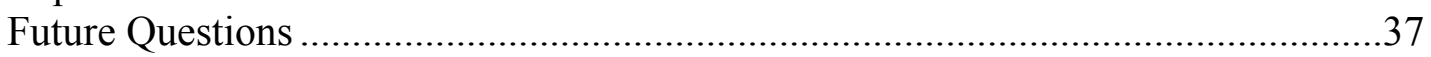

LIST OF REFERENCES......................................................................................................38

APPENDIX A. PARTICIPANT INSTRUCTIONS........................................................43 
APPENDIX B. GROUP DATA ACROSS DUAL TASK CONDITIONS

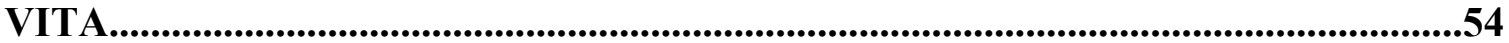




\section{LIST OF TABLES}

Table 2-1. Dual Task and Complex Dual Task Auditory, Visual Accuracy, and Speed Average Scores................................................................................13

Table 3-1. Participant Characteristics: Group 1 - Younger Individuals .........................18

Table 3-2. Participant Characteristics: Group 2 - Older Individuals .............................19

Table 3-3. Experimental Conditions …………………….....................................20

Table 4-1. Dual Task Auditory, Visual Accuracy, and Speed Average Scores .............26

Table 4-2. Comparison of Overall Task Performance of 95\% Confidence Intervals ....28 


\section{LIST OF FIGURES}

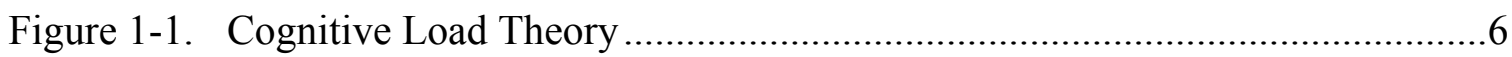

Figure 1-2. Matty's Perceptual and Cognitive Load Model Superimposed over Kirschner's Cognitive Load Theory ................................................................

Figure 3-1. Mean Four Frequency Pure Tone Average (PTA) for the Right and Left Ears for Groups 1 and 2

Figure 4-1. Pairwise Means in Auditory Accuracy Task Performance ...........................29

Figure 4-2. Pairwise Means in Visual Accuracy Task Performance ………………........30

Figure 4-3. Pairwise Means in Processing Speed Task Performance................................31

Figure B-1. Group 1 (Younger) and Group 2 (Older) Auditory Accuracy during Simple Dual Task

Figure B-2. Group 1 (Younger) and Group 2 (Older) Visual Processessing Accuracy during Simple Dual Task

Figure B-3. Group 1 (Younger) and Group 2 (Older) Visual Reaction Time during Simple Dual Task

Figure B-4. Group 1 (Younger) and Group 2 (Older) Auditory Accuracy during Moderate Dual Task

Figure B-5. Group 1 (Younger) and Group 2 (Older) Visual Processing Accuracy during Moderate Dual Task

Figure B-6. Group 1 (Younger) and Group 2 (Older) Visual Reaction Time during Moderate Dual Task

Figure B-7. Group 1 (Younger) and Group 2 (Older) Auditory Accuracy during Complex Dual Task.

Figure B-8. Group 1 (Younger) and Group 2 (Older) Visual Processing Accuracy during Complex Dual Task

Figure B-9. Group 1 (Younger) and Group 2 (Older) Visual Reaction Time during Complex Dual Task 


\section{CHAPTER 1. INTRODUCTION}

\section{Background}

The baby boomer population has now begun to enter into retirement, yielding the largest increase in individuals over the age of 65 in US history (United Nations, 2012; Wiener and Tilly, 2002). As of 2011, 35 million individuals were 65 years or older (Pirkl, 2009) and a projected 77 million people will enter into retirement over the next 18 years. Never before has there been such a significant increase in the older population or in individuals aging into the latest decades of life expectancy (United Nations, 2012; Wiener and Tilly, 2002). Overall, health decline with age is a concern across all generations, and it is a prevalent concern within the baby boomer population. One of the most common difficulties reported by normally aging adults is difficulty understanding speech in noise (Anderson, 2011), even in the presence of normal peripheral auditory thresholds. The factors that contribute to reduced performance in noise are not well understood perhaps because hearing and cognitive processes both naturally decline with age, creating challenges for individuals in important areas of daily functioning, such as when communicating and performing tasks of daily living.

Currently, how cognition interacts with auditory speech perception during multitasking is not well understood. This presents a major challenge for rehabilitation specialists who want to address the needs of this ever-growing population. Thus, researchers have begun examining how cognitive decline that accompanies normal aging may be an additional contributing factor to poor speech understanding in noise. One method of investigating the interaction of noise and cognition is by examining how dual task processing (multi-tasking) affects speech perception in noise. The present review will briefly address the effect of aging on cognition and hearing as well as the importance of dual task paradigms.

\section{Cognition}

Cognition was originally described as "the processes by which the sensory input is transformed, reduced, elaborated, stored, recovered and used" (Neisser, 1967, p.4). Although considered a broad definition, it is still used today (Duncan, 2007; Hargie, 2006). Cognition includes cognitive processes such as attention, memory, executive function, language, and visuospatial skills. Cognitive processes are essential for everyday functioning during both simple and complex tasks; however, cognitive functioning during activities of everyday living is poorly understood. "Wait, where are my keys?" "Now, why did I come into this room?" "Can you repeat that again?" These phrases represent frequent situations of "memory lapse" experienced by countless individuals. In many cases, these occurrences represent a breakdown in memory due to the overload of multitasking during the day. As individuals age, multitasking becomes increasingly affected primarily due to natural changes in working memory (Baddeley, Kopelman, and Wilson, 2002). Multitasking usually involves multimodal processes. That is, input from 
the visual and auditory system are usually being processed while a person is concentrating on a task. Hence, it is important to understand how sensory and cognitive processes interact in multitasking.

\section{The Effect of Age on Cognition and the Brain}

Cognitive function begins to show predictable but varied rate of decline with age (Salthouse, 2009). Beginning at age 25, individuals start to demonstrate difficulty with arithmetic and processing speed (Birren, 1996). In subsequent years, individuals begin to exhibit decline in memory ( $30 \mathrm{~s}-60^{\text {th }}$ decade), reasoning, verbal ability and visuoperception skills (50-60 ${ }^{\text {th }}$ decade), and working knowledge, vocabulary, and word reading $\left(70^{\text {th }}+\right.$ decade $)$ (Birren, 1996). These changes include alterations to cognitive flexibility, the working memory subcomponent of memory, and speed of processing (Giovanello and Verfaellie, 2001, Hedden and Gabrieli, 2004). Cognitive flexibility refers to the ability to adapt to a situation or problem solve in a novel or unique way. Cognitive flexibility engages various cognitive skills, including executive function and working memory. Working memory is defined as the online processing of known and newly learned materials (Baddeley, Kopelman, and Wilson, 2002). Working memory holds multiple pieces of information from various resources combining and manipulating the information for output integration. Memory is an important cognitive skill used during daily living. The prefrontal cortex (PFC) has been linked as "the neural substrate" of working memory (Muller, 2006, p. 53), and is lateralized to the left hemisphere in young adults. It is commonly understood that generalized neuroanatomical atrophy occurs with normal aging processes. Degeneration of neuronal processes occurs in white matter, affecting cortical and, more specifically, subcortical structures (Rabbit et al., 2007; Reuter-Lorenz, 2010; Wong, 2010;). This atrophy and degeneration disrupts and slows the speed of neurophysiological signal processing in the brain, and it may be associated with slower behavioral processing speeds. Therefore, the normal neuroanatomical changes that occur with age negatively affect neurophysiology and, consequently, individual performance, for auditory and cognitive function.

Recent neuroimaging studies (Cabeza et al., 2002; Davis et al., 2008; Getzman, 2011; Peelle et al., 2010) in conjunction with behavioral and EEG (Getzman, 2011) studies, suggests that older adult's compensate for the age related declines in neural structures through the recruitment of different neural networks when compared to younger adults. Specifically during working memory tasks young adults show activation of the left prefrontal cortex (PFC), primarily. Older adults, however, show bilateral activation while performing the same tasks as younger adults. This pattern of activation in older adults is referred to as HAROLD (Hemispheric Asymmetry Reduction in Old Adults). Researchers have recently begun exploring the reasons for the HAROLD pattern in older adults and have proposed a hypothesis for the pattern, known as the decline compensation hypothesis (Cabeza, 2002). The decline-compensation hypothesis stipulates that in older adults general brain area activation will occur in order to compensate for cortical and sub-cortical areas of decline, particularly in sensory processing (Wong, 2010). Thus, according to the decline-compensation hypothesis the 
older brain will recruit additional neural networks to preserve or improve performance on tasks, relative to younger adults. Evidence for this is provided more recently by Wong et al, (2009) who studied spoken language processing in the context of multi-talker babble in older and younger adults. In their study, older adults showed reduced neural activation in the auditory cortex, but increased activation in the prefrontal cortex. It is suggested that older adults compensate for age related declines with increased recruitment of more general cognitive areas.

\section{Effect of Aging on Hearing}

As mentioned previously, hearing also naturally declines with age, leading to a condition called presbycusis. Presbycusis is one of the most common types of hearing loss of older individuals (NIDCD, 1997). It is ranked as the "third most prevalent chronic condition in older Americans" (Yueh, 2003, p. 1976). The prevalence of presbycusis increases with age (Brant, 1990), accounting for the following statistics in the American population: $25 \%-40 \%$ in 65 years old, $40 \%-66 \%$ in 75 years old, and $80 \%$ in $85+$ years

old. (MedlinePlus, 2012; Raynor, 2009; Yueh, 2003, p. 1976). This number is expected to rise with increased exposure to noise in the environment and prolonged lifelong exposure to noise with societal changes. Recent research showed that hearing loss for frequencies important for speech perception $(500 \mathrm{~Hz}-8000 \mathrm{~Hz})$ increased at an average rate of 0.91 $\mathrm{dB} /$ year over an eleven-year period for older adults (Kiely, 2012). Further, Brant et al. (1990) conducted a longitudinal study investigating hearing loss across age groups. Results indicated change in performance across frequencies with age. Additionally, Gates et al. (1991) examined the difference in change of threshold levels across frequency loss. The findings supported earlier results (Brant, 1990) for change across frequencies, particularly higher frequencies for older individuals (Gates, 1991).

Today, it is widely agreed that the primary cause of presbycusis is due to peripheral pathology, that is, degeneration of the peripheral auditory system, including the inner and outer hair cells of the cochlea, the stria vascularis (blood supply to inner ear), and other structures of the inner ear and auditory nerve (Gates, 2005; Humes, 1996; Pichora-Fuller, 2006; Shucknect, 1964). Risk factors for the development of presbycusis include: diabetes, cardiovascular disease, and high blood pressure (Austin, 2009; Cheng, 2009; Gates, 1993). These are diseases that may disrupt blood supply to inner ear and auditory nerve and are frequently observed in the elderly. Additionally, central presbycusis is thought to occur as a result of degraded central auditory processing, due to degradation in the auditory cortex and midbrain, in normal aging (Humes, 2012). Central processing challenges result in difficulty understanding in noise. Thus, a person may have essentially normal auditory thresholds (normal peripheral function) but exhibit significantly degraded performance in noise (central auditory processing). It is thought that presbycusis may begin as a peripheral problem, but it may reflect a combination of both peripheral and central mechanisms as individuals age (Gates, 2009).

Researchers have started to investigate the contribution of cognitive processing and auditory processing, particularly in noise. Kiely, Gopinath, Mitchell, Luszez, and 
Anstey (2012) found that cognitive decline was related to decline in hearing function. They examined cognitive, health, and sociodemographic factors in 3,526 individuals over 11 years. Participants were drawn from the Australian Longitudinal Study of Ageing (ALSA) and the Dynamic Analyses to Optimize Ageing (DYNOPTA) project. Audiometric and cognitive assessments were performed to record hearing and cognitive function. Detailed survey and medical history records were compiled into health and sociodemographic information. Assessments and surveys were conducted during 5 phases of the 11 year data collection period. Kiely et al. (2012) found that the rate of change of hearing over time was due to: age, gender, noise exposure in the workplace, and the presence of cognitive decline. Specifically, results indicated that cognitive decline was related to elevated pure tone averages (PTA), the average of hearing thresholds at $500 \mathrm{~Hz}$, $1000 \mathrm{~Hz}$, and $2000 \mathrm{~Hz}$. These results support the assertion that the relationship between cognitive function and hearing, though not well understood, is interdependent. Interestingly, Keily et al. (2012) also noted a hearing pattern decline similar to that of cognitive decline patterns for gender. Cognitive and hearing decline occur gradually over time for men whereas women experience a faster decline over a shorter period of time. Through this longitudinal study, Keily et al. (2012) have added to the growing body of literature linking the relationship of hearing and cognitive function.

Recent research has begun examining cognitive processing and auditory processing in older listeners. For example, Getzmann et al. (2011) examined neurological correlates of speech perception under demanding listening conditions using electrophysiological (EEG) and behavioral measures. Specifically, researchers were interested in investigating speech perception changes in young and older adults, under demanding listening conditions. In their experiment, listeners were asked to attend to stock prices, and respond when a pre-specified stock hit a certain price. In this experiment, listeners were asked to ignore irrelevant stocks and prices, as well as beeps that were randomly presented. EEG results suggested reduced brain activation in the parietal lobe for older individuals compared to younger individuals (Getzmann et al., 2011). Furthermore, a combination of behavioral and EEG measures suggest that for older individuals, successful performance during demanding listening situations correlated with increased activation of the frontal lobe areas of the brain (Getzmann et al., 2011). Researchers concluded that the increased brain activation in the frontal lobes support "allocation of mental resources for compensation of potential declines in spoken language understanding" (Getzmann et al., 2011, p. 8). Additionally, Getzmann et al. (2012) stipulated that the resource allocation during demanding listening conditions is compensation for central processing due to age related differences. Hence, changes in neuroanatomical structures contribute to neurophysiological alterations in hearing and cognitive processing which can affect speech perception. This finding is consistent with the decline compensation hypothesis.

\section{Perception and Cognitive Load Model}

Cognitive load is the amount of cognitive processing utilized while performing a task or group of tasks at one time (Haga, 2002, Young, 2008). Traditionally perceptual 
factors have not been considered in models of cognitive load. For example, Figure 1-1 shows the Kirschner et al. (2002) cognitive model without perception as a separate factor. Cognitive load theories attempt to account for the factors that influence cognitive load during task performance. Models of cognitive load typically divide these factors into intrinsic and extrinsic/extraneous factors (Kirschner, 2002; Sweller, 1998). Intrinsic factors refer to task characteristics, and extrinsic/extraneous factors are the instruction characteristics. Intrinsic cognitive load is the inherent difficulty or cognitive load related to a task. Extrinsic/extraneous cognitive load is related to the difficulty or cognitive load due to the manner in which the task is given. According to the Cognitive Load Theory, the factors that influence cognitive load fall into three specific categories: task (including environment), learner (individual factors), and task/learner interaction (Kirschner, 2002). Thus, noise is considered a contributor to task but not examined for independent influence. However, recent literature suggests that perception and cognition interact. In order to understanding how perceptual demand and cognitive demand contribute to overall cognitive load, Mattys introduced the Perceptual and Cognitive load Model (PCLM).

Mattys introduced the Perceptual and Cognitive load Model (PCLM) in order to understand the factors which add to overall cognitive load, suggesting that perceptual load is a significant contributing factor to overall cognitive load. Specifically, Mattys et al. (2009) define perceptual load as " any alteration to the signal leading to diminished acoustic integrity". According to Mattys perceptual load can contribute to cognitive load when it leads to the recruitment of central processing resources due to concurrent attentional and mnemonic processing. For example, when listening under difficult conditions, perceptual load increases, and mental resources are allocated or re-allocated to meet the demands of the task. To summarize, Mattys suggests that perceptual load is a component of cognitive load and should be considered in future auditory research.

According to Mattys et al. (2009), noise can result in two types of masking, known as energetic masking and informational masking. Energetic masking is noise that decreases audibility of the speech signal, thereby reducing the intelligibility of the message. Thus, perceptual decline in performance can occur due to energetic masking alone. Additionally, Mattys et al. (2009), described a second type of performance decline known as informational masking. Informational masking results in unintelligible speech due to the level of task complexity and disruption to the acoustic signal. The portion of disruption due to task complexity when noise has been accounted for is the recognized informational masking. (p.6) Finally, Mattys et al. suggests that informational masking is the link to understanding "the effect of perceptual and cognitive loads on speech recognition" (p.34). See Figure 1-2 for an illustration of how Mattys Perceptual and Cognitive Load Model might be applied to Kirschner's Cognitive Load Theory. If considered together, the Mattys Model and Kirschner Theory account for interrelated cognitive and perceptual loads. The Figure 1-2 shows perceptual load added to influential factors of cognitive load. For example, informational masking (perceptual) is added as a contributing factor to intrinsic cognitive load (load due to task). Where as, energetic masking (perceptual) would be a factor of extrinsic or extraneous cognitive load 


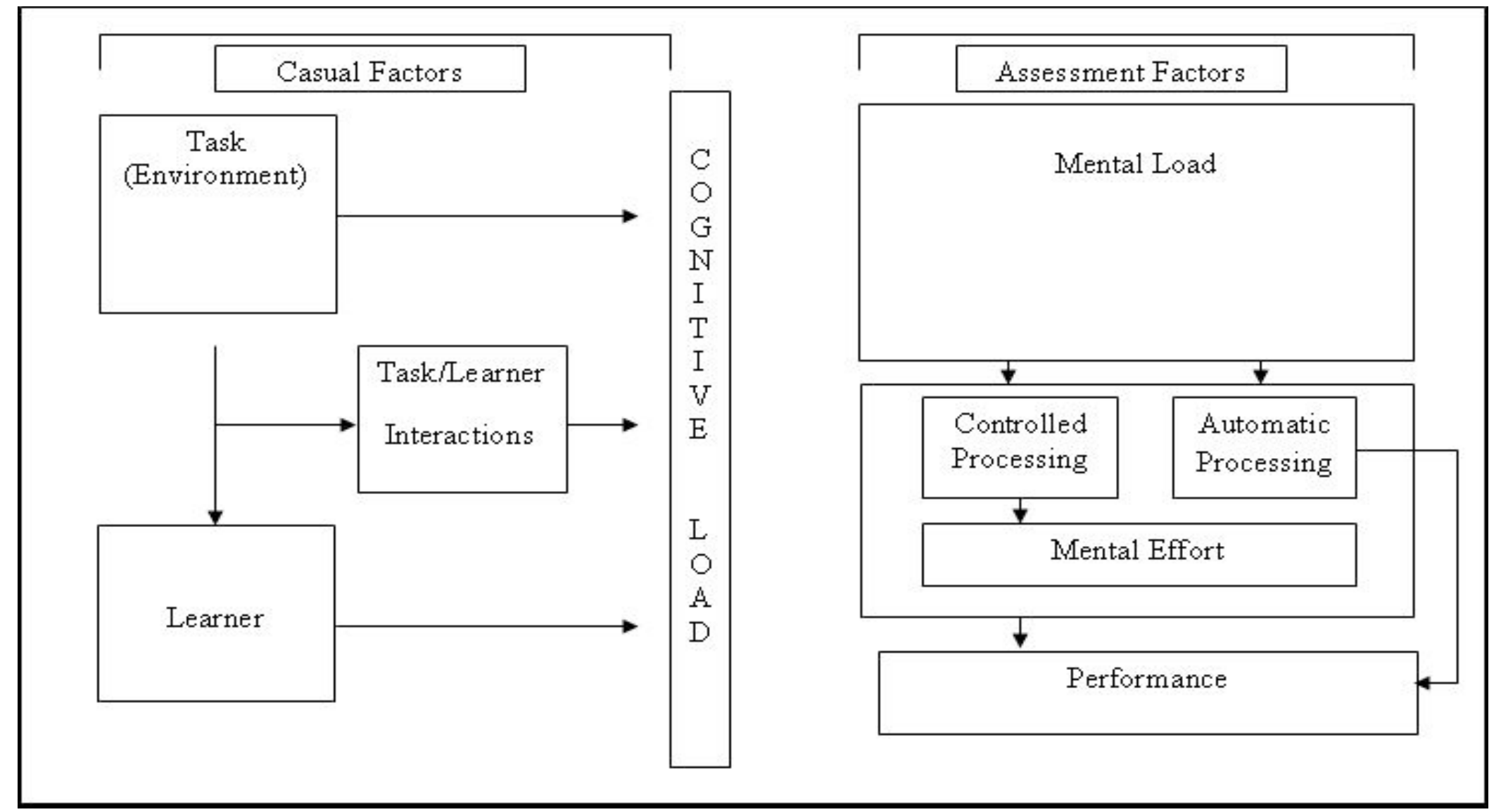

Figure 1-1. Cognitive Load Theory

Reprinted with permission. Kirschner, P. (2002). Cognitive load theory: Implications of cognitive load theory on the design of learning. Learning and Instruction, 12, 1-10. 


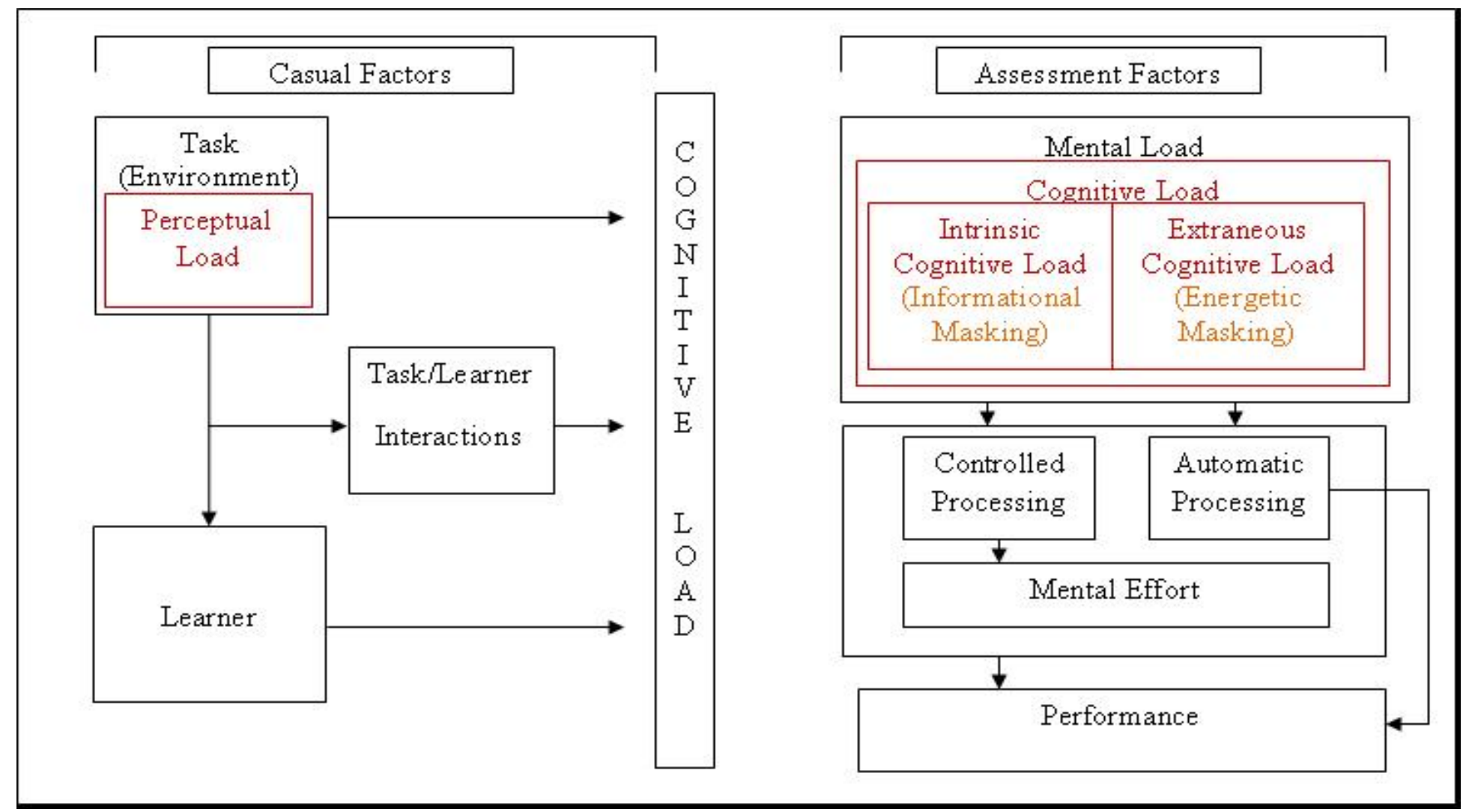

Figure 1-2. Matty's Perceptual and Cognitive Load Model Superimposed over Kirschner's Cognitive Load Theory

Modified with permission. Kirschner, P. (2002). Cognitive load theory: Implications of cognitive load theory on the design of learning. Learning and Instruction, 12, 1-10. 
(load due to instruction of task). In other words, for each of the subtypes of cognitive load there is a parallel perceptual load factor.

To summarize, as individuals age, hearing and cognitive function decline. Neuroanaomtical and physiological changes occur with age, yielding a range of patterned decline in cognitive function. Similarly, anatomical and physiological alterations lead to degeneration of the peripheral auditory system, yielding presbycusis. Recent research has begun examining cognitive processing and auditory processing together in older listeners. The Perceptual and Cognitive Load Model (PCLM) incorporates perceptual load as a contributing factor to overall cognitive load. Mattys et al. (2011) suggests that the key to understanding speech recognition is through the study of informational masking which is examined using Dual-task paradigms (DTP). DTP, that systematically exert different levels of cognitive and perceptual load on the system, can provide additional understanding of the effect of cognitive load on speech recognition (noise and task). Thus far, researchers have not applied this model to speech perception in older individuals. Overall, little research has been conducted using dual task paradigms and speech perception in noise in aging individuals. The following literature review will focus on recent literature investigating dual task paradigms in speech perception in noise. 


\section{CHAPTER 2. LITERATURE REVIEW}

\section{Cognition and Noise}

The dual task paradigm (DTP) is a sensitive tool for the examination of cognitive load and is utilized in cognition and noise research (Gosselin, 2010; Holtzer, 2005). Dual Task paradigms include primary and secondary tasks administered first separately and then concurrently (Gosselin, 2010). Researchers initially began to examine listening effort by considering how auditory noise affects auditory accuracy on both a primary and secondary task. While individuals can maintain auditory accuracy for a primary task, there is diminished performance when a secondary task is added. The difference in performance of the secondary task is considered an index of listening effort, whereas, the total effort expended during a task or set of tasks is the cognitive load. Although load during dual task is better understood for basic, or easy dual task conditions, higher-level or more complex dual tasks representative of everyday life and the effects of noise, are not well understood. Very few studies have implemented dual tasks paradigms to examine speech in noise in older adults (Gosselin, 2010; Sarampalis, 2009).

Pichora-Fuller et al. (1995) examined age-related differences in cognition and speech perception in participants ages 19-91 year olds with differing hearing function. Specifically, they examined the effect of noise on auditory and visual processing. They performed two experiments utilizing the revised Speech Perception in Noise (SPIN) test (Bilger, 1984). During experiment one, participants were asked to listen to high and low context sentences and identify the final word in the sentence, at several SNRs. This was a traditional speech perception in noise task with no additional dual task. Results showed that younger listeners achieve maximal advantage at poorer SNRs than older listeners. Additionally, results showed a difference in high/low context performance due to age. Specifically, older individuals demonstrated greater benefit from context when compared to the younger group. Researchers suggested that older individuals may be relying more on cognitive domains to complete the same tasks as younger individuals. This finding supports the decline compensation hypothesis. In experiment two, subjects were asked to listen to sentences, indicate if the sentence had context (primary task), hold it in memory, while listening to the next sentence, and recall the final words in the sets of sentences (secondary task) (working memory-dual task) after all sentences in a set had been heard. The SPIN sentences were modified for the working memory task and were examined at quiet, 0,5 , and $8 \mathrm{~dB}$ SNR, within four list sets of 2, 4, 6, and 8 sentence sequences. The sentence sequences were implemented for evaluating the range of working memory capabilities in different noise conditions. Findings suggested that set size affected word recall performance during the least challenging listening condition. There was a performance difference noted due to age and SNR. Specifically, younger individuals performed better than older individuals during recall performance for a set size of four, six, and eight items. Further, there was a significant difference in performance between listening conditions at 0dB SNR and 5dB SNR or a set size of four, six, and eight items. An age difference was observed for set size according to listening conditions. Specifically, recall performance for set size was related to listening condition 
when researchers compared. Researchers found a significant difference between older and young individuals as well as significant difference between 5 and $0 \mathrm{~dB}$ SNR listening conditions. Therefore, this result indicates a level of change at 5 and $0 \mathrm{~dB}$ SNR across both groups. Finally, in order to investigate whether the reduction in word recall observed in older individuals was due to general aging effects on the auditory system or to general cognitive declines affecting all modalities, an additional experiment was performed, for word recall of read sentences. Thus, they compared the word recall of heard sentences to that of read sentences. Findings indicated modality performance differences with age. Younger participants scored better than older participants for auditory recall, whereas, both the older and younger participants performed similarly during the read recall task. These results suggested that the declines observed in recall in the previous experiment are more likely due to age related differences in auditory processing, rather than general aging effects affecting all recall. Overall, study results showed that : (1) as noise levels became more challenging, cognitive function, even at the single task level, declined; (2) more challenging noise environments affected all participant cognition regardless of age or cognitive function; and (3) changes of auditory processing across age negatively affect auditory word recall. Pichora-Fuller et al, suggested that these results support a processing model in which cognitive resources are used to support auditory processing for challenging listening situations or because of age related declines in auditory processing. These results support the Perceptual and Cognitive Load Model for the relationship between perceptual load and cognitive load.

One of the goals of the following study was to establish a dual task paradigm for the investigation of cognitive load during speech-in-noise conditions. Sarampalis et al. (2009) studied the effect of noise reduction on cognitive load during dual task in 18-26 year olds with normal hearing function. The dual task activities included word recognition, working memory, and visual processing speed. Specifically, they tested the hypothesis that noise reduction processing reduced cognitive effort when listeners are engaged in dual tasks involving speech perception and working memory or speech perception and visual processing. Researchers administered two experiments, utilizing the SPIN sentences. During experiment one, participants were asked to repeat the last word in sentence (word identification-primary task) and then produce the word during later recall (working memory — secondary task) under differing signal to noise ratio SNR (quiet, $-2,+2 \mathrm{~dB}$ ). They tested this under an unprocessed condition and in a processed condition that approximated noise reduction in hearing aids. In that experiment they measured the percent of words correctly identified as well as the percent of words correctly recalled (cognitive load) in noise (perceptual load) during dual task. Results showed that activation of noise reduction did not result in improvements in speech perception at poor SNRs, but resulted in increased percentage of words recalled, relative to the unprocessed condition at poor SNRs. In a second experiment with a different group of listeners, Sarampalis et al. examined a second type of dual task assessing speech perception (in quiet and noise) while performing a timed complex visual task (cognitive task). Participants were asked to repeat the sentence (primary task), while concurrently completing a visual processing speed task (secondary task) under differing signal to noise ratio SNR (quiet, $-6,-2,+2 \mathrm{~dB}$ ) in the processed and unprocessed condition. Findings indicated that processing speed decreased (got faster) as noise conditions improved; 
conversely, as noise levels worsened, processing speed increased or slowed down. Sarampalis et al. (2009) found that better SNRs result in improved performance in dual task dyads of memory and processing speed, reaffirming previous findings that the background noise in the auditory environment can negatively affect listening effort and cognitive performance specific to young listeners. Further, these findings do support use of noise reduction to release cognitive resources for reallocation. It is further argued that the greatest value of noise reduction may not be for improved hearing but as a cognitive aid resulting in reduction of cognitive load.

Pichora-Fuller et al. (1995) and Saramplis et al. (2009) provide an initial framework for the investigation of the effects of noise on cognitive load and cognitive function. During both research studies a systematic investigation of cognitive skills was not conducted a priori to establish normal cognitive function in their sample participants. This is necessary to provide a standard baseline of cognitive function across all participants. Determining a baseline of cognitive function for each participant ensures that performance during the experiment is representative of a specified group (i.e. normal cognitive aging or dementia).

Pichora-Fuller et al. (1995) and Saramplis et al. (2009) employed dual task paradigms; however, the DTPs they used were basic, and did not systematically challenge the participant. During both studies, the isolated and dual task combinations were not conducted in a systematic progression. That is, they studied some tasks in isolation and dual task combinations, but did not apply a systematic methodology that would allow analysis of each cognitive skill set. Analysis of this change would increase understanding of the relationship between cognitive skill and speech in noise performance. Further, Saramplis et al. (2009) included a task examining processing speed designed with visually alternating fields, eliciting interhemispheric processing, a potential confound to the study of cognitive processing speed because interhemispheric transfer time could impact the measured processing speed. Finally, conclusions drawn regarding the effects of decreased SNR conditions on cognitive function are degraded by lack of establishment of participant cognitive function at baseline, in both studies. In review of similar speech in noise studies, the SNR levels may not have ranged significantly enough to represent truly varied SNR environments, and, if altered to pinpoint more challenging SNR levels, the study may potentially yield clearer results. Diverse or more challenging SNR levels may better represent environments of everyday life producing more applicable study results.

\section{Preliminary Study}

In order to address systematic examination of tasks in isolation and dual task, establishment of baseline cognitive data, and processing speed task limitations in the above studies, Harvey et al. (in preparation) investigated the effects of noise on cognitive

function in normally aging adults. The purpose of that study was to test the effect of noise on cognitive function in increasingly difficult tasks that are thought to systematically increase cognitive load. The study focused on the following tasks: simple task (auditory 
only and visual only), dual task (visual processing + word recognition), and complex task (visual processing + working memory in noise). Specifically, we wanted to test if SNRs differentially affect cognitive function, in single, dual, and complex task paradigms. We hypothesized the following: participants would demonstrate decreased cognitive skill (processing speed, word recognition, and working memory) with increased task complexity and increased difficulty in SNR. Specifically, within tasks, it was hypothesized that participants would demonstrate poorer cognitive processing under reduced/difficult SNR conditions. Thus, we expected that noise would negatively affect auditory accuracy and reaction time measures. Additionally, we hypothesized that participants would demonstrate decreased auditory accuracy as task complexity increased from tasks in isolation to dual task paradigms. Fifteen right-handed adults, ages 65-80 years, participated. All participants were tested in each of 4 experimental conditions (1) word recognition (Simple Auditory only), (2) visual processing (Simple Visual only), (3) auditory word recognition + visual processing (Dual Task), and (4) auditory working memory and visual processing (Complex Dual Task) in noise, under various SNR conditions. Response auditory accuracy and reaction times were measured. An overview of the results is provided in Table 2-1.

Results of the auditory accuracy analyses for auditory tasks suggest that task complexity and noise significantly affect auditory accuracy for both auditory word recognition (Simple and Moderate Dual Task) and working memory (complex dual task) cognitive skills. These findings support the hypothesis that cognitive function is affected by noise and various levels of cognitive load. Visual processing accuracy results differed from auditory accuracy, suggesting that task, not noise, significantly affects visual processing accuracy. Additionally, the processing speed analyses suggest that as tasks become more complex speed of performance is significantly affected. It was hypothesized and confirmed that as a task becomes more complex (Simple and Dual Task) speed slows down. Whereas, in more complex dual task conditions, it was noted that performance did not necessarily follow the same pattern but remained inversely affected by concurrent task conditions. The findings suggested that individuals presented with tasks of high complexity or tasks under $+5 \mathrm{~dB}$ SNR of noise or greater will demonstrate significant decline in performance compared to less challenging task conditions because hard tasks and tasks with a lot of noise are significantly more challenging compared to similar tasks of easier complexity or noise conditions. The range of auditory accuracy scores in the complex condition suggests that this task was very difficult for participants regardless of noise condition. Hence, the Complex Dual Task may represent a task that is more challenging than those that are representative of daily living. Furthermore, addition of a task that targets moderate complexity may provide a complex task that represents challenges in daily living.

Given the fact that the Cognitive Load Theory suggests that individual factors such as age are thought to contribute to cognitive load, it is uncertain whether age is a confound in these results. The participant group in our study had a broad age range of $65-$ 80 years. Because the literature suggests that early presbycusis is primarily of a peripheral rather than central nature, it may be the case that individuals of different age groups may show different effects of noise and cognitive load on cognitive function. 
Table 2-1. Dual Task and Complex Dual Task Auditory, Visual Accuracy, and Speed Average Scores

\begin{tabular}{|c|c|c|c|c|c|c|}
\hline \multirow[b]{2}{*}{$\begin{array}{l}\text { Listening } \\
\text { Condition }\end{array}$} & \multicolumn{3}{|c|}{ Dual Task } & \multicolumn{3}{|c|}{ Complex Dual Task } \\
\hline & $\begin{array}{l}\text { Auditory } \\
\text { Accuracy }\end{array}$ & $\begin{array}{c}\text { Visual } \\
\text { Accuracy }\end{array}$ & $\begin{array}{c}\text { Visual Processing } \\
\text { Speed }\end{array}$ & $\begin{array}{l}\text { Auditory } \\
\text { Accuracy }\end{array}$ & $\begin{array}{c}\text { Visual } \\
\text { Accuracy }\end{array}$ & $\begin{array}{c}\text { Visual Processing } \\
\text { Speed }\end{array}$ \\
\hline Quiet & $99 \%$ & $99 \%$ & $\begin{array}{c}524.42 \\
(\mathrm{SD}=40.38)\end{array}$ & $26 \%$ & $96 \%$ & $\begin{array}{c}750.90 \\
(\mathrm{SD}=81.96)\end{array}$ \\
\hline $5 \mathrm{~dB}$ & $93 \%$ & $99 \%$ & $\begin{array}{c}558.67 \\
(\mathrm{SD}=12.66)\end{array}$ & $25 \%$ & $91 \%$ & $\begin{array}{c}834.90 \\
(\mathrm{SD}=27.29)\end{array}$ \\
\hline $0 \mathrm{~dB}$ & $83 \%$ & $99 \%$ & $\begin{array}{c}557.44 \\
(\mathrm{SD}=39.35)\end{array}$ & $14 \%$ & $98 \%$ & $\begin{array}{c}769.93 \\
(\mathrm{SD}=294.57)\end{array}$ \\
\hline
\end{tabular}


Thus, examination of multiple groups of adults may give a greater indication of age affects for the effect of noise on cognitive function during cognitive load tasks.

\section{Current Study}

The Cognitive Load Theory and the Perceptual and Cognitive Load models will be utilized to examine the robustness of cognitive processing in noise during dual task processing in young and older adults.

Question 1: Does Participant Age (casual factor) differentially affect cognitive function, in dual task paradigms (cognitive load)? The hypothesis predicts that participants for both younger and older groups will exhibit performance decline, with the older group demonstrating a more significant decline of cognitive skill with increased task complexity and/or increased difficulty in SNR. Specifically, the younger group will demonstrate more tolerance of noise during dual tasks than older individuals.

Additionally, younger individuals will exhibit higher auditory or visual accuracy and increased processing speed (cognitive load) when compared to older individuals, in challenging listening conditions (perceptual load). The CLT predicts that factors such as age would affect performance. If a group difference is found this may support the CLT.

Second, given that Sarampalis and Pichora-Fuller did not make their dual tasks more challenging in a systematic way, it is unknown how task complexity and perceptual load interact during speech perception in noise. Thus, Question 2 of the study was: Do signal-to-noise ratio (perceptual load) or Task complexity (cognitive load) differentially affect cognitive function, in dual task paradigms? The Perception and Cognitive Load Model asserts that SNR contributes to the environment within the context of Task and that task complexity does influence performance. The hypothesis predicts that participants will demonstrate decreased cognitive skill with increased task complexity and/or increased difficulty in SNR. Specifically, (1) with each progressive increase in cognitive load (task), participants would demonstrate decreased auditory or visual accuracy and slower reaction times; (2) with each progressive increase in perceptual load (noise), participants would demonstrate decreased auditory or visual accuracy and slower reaction times. The results of the preliminary study suggested that the mid condition may show an interesting pattern in performance within subjects. Thus, specific predictions are made regarding the mid condition for noise and cognition.

- Question 2a: Does the 3 SNR (mid-condition, perceptual load) differentially affect cognitive function in dual task paradigms? The hypothesis predicts that participants will demonstrate cognitive skill significantly different than at other SNR conditions.

- Question 2b: Does the Moderate Dual Task (mid-condition, cognitive load) will differentially affect cognitive function in noise? The hypothesis predicts that participants will demonstrate cognitive skill significantly different than at other dual task conditions. 
In summary, the hypotheses predicted: (1) participants for both younger and older groups would exhibit performance decline (with the older group demonstrating a more significant decline) of cognitive skill with increased task complexity and/or increased difficulty in SNR; (2) with each progressive increase in cognitive load (task), participants would demonstrate decreased auditory or visual accuracy and slower reaction times; and (3) with each progressive increase in perceptual load (noise), participants would demonstrate decreased auditory or visual accuracy and slower reaction times. 


\section{CHAPTER 3. METHODOLOGY}

\section{Participants}

Twenty-nine native speakers of American English participated. The subjects were divided into two groups based upon age. Group 1 consisted 14 listeners (Female=11) who were 40-59 years old (Mean=53.18, $\mathrm{SD}=5.97)$. Group 2 consisted of 15 listeners $($ Female $=9)$ who were 60 years and older $($ Mean=72.07, $\mathrm{SD}=5.11)$. All subjects participated in all three experimental conditions. All participants demonstrated normal to near-normal hearing and normal middle ear function on audiometric assessment. Normal to near-normal hearing criterion included thresholds less than or equal to $35 \mathrm{~dB}$ HL from $250 \mathrm{~Hz}-3000 \mathrm{~Hz}$ (Pichora-Fuller, 1995). The Mean 4 frequency pure tone average (PTA) for the right and left ears for Group 1 were $17.18 \mathrm{~dB}$ HL (right), $15.98 \mathrm{~dB} \mathrm{HL}$ (left) and for and Group 2 were $22.67 \mathrm{~dB}$ HL (right), $21.42 \mathrm{~dB}$ HL (left), respectively. The 4-frequency PTA is shown in Figure 3-1. See Figure 3-1, Table 3-1, and Table 3-2 for the demographic characteristics of participants of Group 1 and Group 2, respectively. Volunteers were informed of project protocol, reviewed, and signed consent forms prior to agreeing to participate. This project was approved by The University of Tennessee Health Science Center's Institutional Review Board.

Cognitive function was assessed with a screening battery of standardized cognitive subtests from the Woodcock-Johnson III (2001) test of cognitive abilities including: Memory for Words, Auditory Working Memory, Decision Speed, and Visual Matching, which provided baselines for the basic functions of processing speed and working memory. The inclusion criterion for cognitive function was based upon the standardized measures for each assessment. Participants were required to achieve standard scores greater than 85 on at least three of the four subtests. See Table 3-1 and Table 3-2 for subtest standard scores. Participants exhibited normal or corrected vision (e.g. glasses or contacts) per self-report. Participant handedness was established using the standardized Edinburgh Handedness Inventory, where right handedness $=\mathrm{R}>40$ (Oldfield, 1971). Twenty-six right handed, two ambidextrous, and one left handed participants were included. Participants were recruited from the Knoxville community through fliers and informational handouts.

\section{Procedure}

\section{General Description of Task Conditions}

All participants were tested in each of three experimental conditions, all of which involved a dual task paradigm combining auditory and visual processing in noise. The Simple Dual Task Condition investigated an auditory speech perception and visual processing speed. Moderate Dual Task Condition examined auditory word recall (isolated words) and visual processing. The Complex Dual Task Condition involved auditory 


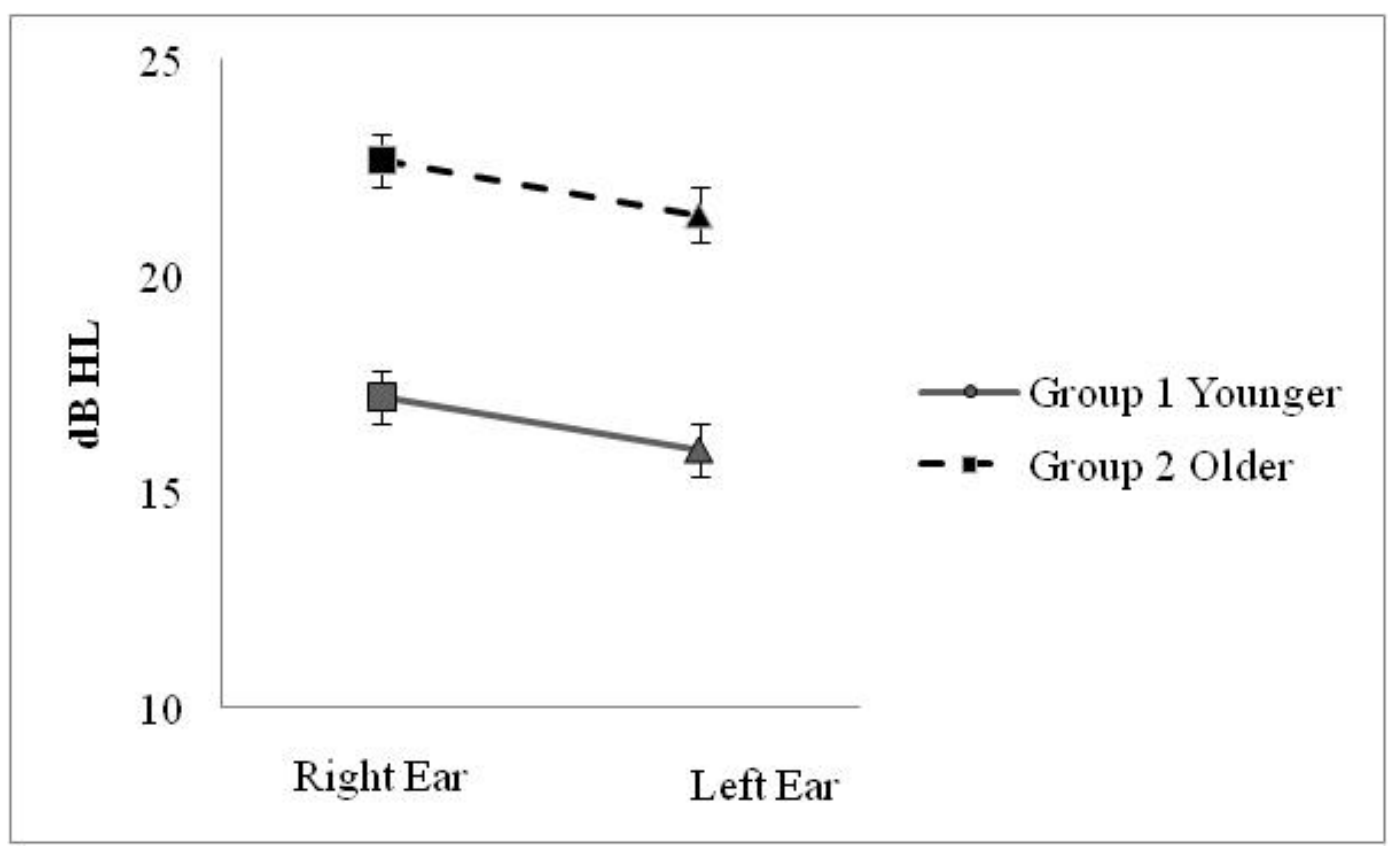

Figure 3-1. Mean Four Frequency Pure Tone Average (PTA) for the Right and Left Ears for Groups 1 and 2 
Table 3-1. Participant Characteristics: Group 1 - Younger Individuals

\begin{tabular}{|c|c|c|c|c|c|c|c|}
\hline \multirow[b]{2}{*}{ Subject } & \multirow[b]{2}{*}{ Age } & \multirow[b]{2}{*}{ Gender } & \multirow[b]{2}{*}{$\begin{array}{l}\text { Hearing R/L } \\
\text { (PTA) }\end{array}$} & \multicolumn{4}{|c|}{ Cognition Screen (Standard Scores) } \\
\hline & & & & $\begin{array}{l}\text { Memory for } \\
\text { Words }\end{array}$ & $\begin{array}{l}\text { Auditory } \\
\text { Working } \\
\text { Memory }\end{array}$ & $\begin{array}{l}\text { Decision } \\
\text { Speed }\end{array}$ & $\begin{array}{c}\text { Visual } \\
\text { Matching }\end{array}$ \\
\hline DS12 & 51 & $\mathrm{~F}$ & $22.5 / 15$ & 124 & 123 & 122 & 124 \\
\hline DS 15 & 57 & $\mathrm{~F}$ & $12.5 / 11.3$ & 106 & 111 & 124 & 112 \\
\hline DS21 & 54 & M & $23.8 / 17.5$ & 105 & 103 & 83 & 97 \\
\hline DS24 & 41 & $\mathrm{~F}$ & $15 / 8.8$ & 102 & 114 & 98 & 115 \\
\hline $\mathrm{DS} 25$ & 49 & $\mathrm{~F}$ & $21.5 / 15$ & 97 & 104 & 121 & 124 \\
\hline DS26 & 54 & M & $23.8 / 30$ & 125 & 136 & 116 & 117 \\
\hline $\mathrm{DS} 27$ & 52 & $\mathrm{~F}$ & $11.3 / 6.3$ & 98 & 110 & 95 & 96 \\
\hline DS28 & 54 & $\mathrm{~F}$ & $15.3 / 18.8$ & 118 & 97 & 124 & 114 \\
\hline DS29 & 50 & $\mathrm{~F}$ & $12.5 / 11.3$ & 97 & 117 & 95 & 119 \\
\hline $\mathrm{DS} 30^{*}$ & 41 & $\mathrm{~F}$ & $11.3 / 8.8$ & 122 & 118 & 117 & 119 \\
\hline DS31 & 49 & $\mathrm{~F}$ & $5 / 12.5$ & 138 & 126 & 100 & 108 \\
\hline DS32 & 56 & $\mathrm{~F}$ & $17.5 / 16.3$ & 98 & 101 & 102 & 109 \\
\hline DS33* & 54 & M & $8.8 / 7.5$ & 98 & 101 & 96 & 89 \\
\hline DS35 & 57 & $\mathrm{~F}$ & $25 / 26.2$ & 94 & 96 & 116 & 112 \\
\hline $\begin{array}{l}\text { Mean } \\
\text { (SD) }\end{array}$ & $\begin{array}{c}53.18 \\
(\mathrm{SD}=5.97)\end{array}$ & $\mathrm{F}=11$ & $\begin{array}{c}17.18 / 15.99 \\
(\mathrm{SD}=6.11,6.59)\end{array}$ & $\begin{array}{c}109.65 \\
(\mathrm{SD}=12.51)\end{array}$ & $\begin{array}{c}110.94 \\
(\mathrm{SD}=10.77)\end{array}$ & $\begin{array}{c}110.29 \\
(\mathrm{SD}=13.27)\end{array}$ & $\begin{array}{c}112 \\
(\mathrm{SD}=10.42)\end{array}$ \\
\hline
\end{tabular}

$*$ ambidextrous, $* *=$ left handed 
Table 3-2. Participant Characteristics: Group 2 - Older Individuals

\begin{tabular}{|c|c|c|c|c|c|c|c|}
\hline \multirow[b]{2}{*}{ Subject } & \multirow[b]{2}{*}{ Age } & \multirow[b]{2}{*}{ Gender } & \multirow[b]{2}{*}{$\begin{array}{l}\text { Hearing R/L } \\
\text { (PTA) }\end{array}$} & \multicolumn{4}{|c|}{ Cognition Screen (Standard Scores) } \\
\hline & & & & $\begin{array}{l}\text { Memory for } \\
\text { Words }\end{array}$ & $\begin{array}{l}\text { Auditory } \\
\text { Working } \\
\text { Memory }\end{array}$ & $\begin{array}{c}\text { Decision } \\
\text { Speed }\end{array}$ & $\begin{array}{c}\text { Visual } \\
\text { Matching }\end{array}$ \\
\hline DS2 & 79 & $\mathrm{M}$ & $26.25 / 35$ & 121 & 124 & 116 & 94 \\
\hline DS3 & 81 & M & $26.25 / 23.75$ & 123 & 128 & 121 & 126 \\
\hline DS6 & 76 & $\mathrm{~F}$ & $20 / 33.75$ & 120 & 114 & 98 & 101 \\
\hline DS7 & 66 & M & $25 / 20$ & 122 & 105 & 113 & 106 \\
\hline DS8 & 73 & M & $20 / 23.75$ & 131 & 129 & 119 & 105 \\
\hline DS9 & 69 & $\mathrm{~F}$ & $17.5 / 17.5$ & 110 & 112 & 114 & 97 \\
\hline DS10 & 67 & $\mathrm{~F}$ & $11.25 / 11.25$ & 109 & 118 & 133 & 129 \\
\hline DS11 & 70 & $\mathrm{~F}$ & $20 / 18.75$ & 117 & 114 & 89 & 101 \\
\hline DS14 & 73 & $\mathrm{~F}$ & $30 / 17.5$ & 125 & 122 & 133 & 116 \\
\hline DS16 & 69 & $\mathrm{~F}$ & $16.3 / 16.3$ & 123 & 115 & 126 & 113 \\
\hline DS18 & 77 & $\mathrm{~F}$ & $20 / 16.3$ & 113 & 115 & 130 & 132 \\
\hline DS19 & 67 & M & $20 / 18.8$ & 109 & 116 & 122 & 122 \\
\hline DS23 & 77 & $\mathrm{~F}$ & $36.2 / 32.5$ & 121 & 126 & 144 & 147 \\
\hline DS36 & 63 & $\mathrm{~F}$ & $26.3 / 27.5$ & 108 & 116 & 116 & 111 \\
\hline $\mathrm{DS} 37 * *$ & 74 & $\mathrm{M}$ & $25 / 18.75$ & 131 & 128 & 120 & 119 \\
\hline Mean & 72.07 & & $22.67 / 21.42$ & 118.87 & 118.8 & 119.6 & 114.6 \\
\hline (SD) & $(\mathrm{SD}=5.11)$ & $\mathrm{F}=9$ & $(\mathrm{SD}=5.89,7.62)$ & $(\mathrm{SD}=7.37)$ & $(\mathrm{SD}=6.78)$ & $(\mathrm{SD}=13.21)$ & $(\mathrm{SD}=14.26)$ \\
\hline
\end{tabular}

$*$ ambidextrous, $* *=$ left handed 
working memory of words presented in sentence context and visual processing. Each experiment was designed to represent a hierarchical increase in skill set complexity. The Simple Dual Task is considered to be simple because it relies on word recognition of a final word in a sentence while performing a visual task. This task requires the participant to identify a word immediately after hearing it; this is considered an easy recall task. The Moderate Dual task was a more difficult task than the Simple Dual Task because it requires that the listener hold word in memory before repeating them, eliciting word recall. Finally, the Complex Dual Task was considered to be more complex than the Moderate task because it required that the participant remember sets of words that were presented in sentences as opposed to remembering words in isolation. The increasing task difficulty was not systematically examined prior to the experiment. Such that this dual task hierarchy is based upon current literature but has not been methodologically established. These methods are a best guess at achieving hieratical complexity, but not a verified systematic process. Additionally, within each of the experiments complexity was also manipulated through manipulation of the signal-to-noise ratio. Thus, each of the dual task conditions were presented at the following signal to noise ratio (SNR) conditions: 5, 3, and $0 \mathrm{~dB}$ SNR. Table 3-3 shows a breakdown of the experiments. In alignment with the preliminary study, the easiest signal-to-noise ratio (SNR) was $5 \mathrm{~dB}$ with the most challenging noise level at $0 \mathrm{~dB}$. In effect, the complexity of each of the dual tasks was achieved through manipulation of the auditory task, as the complexity of the visual task did not change. The order of experimental condition received by participants was randomized for each participant. Consequently, some participants may have begun the experiment in the Simple Condition, while others started in the Moderate or Complex Dual Task Conditions.

\section{Auditory Task: Stimuli}

The tape-recorded SPIN-R materials (Bilger, 1984) were used for the Simple Dual Task and Complex Dual Task. The SPIN-R materials consisted of eight recorded lists of 50 sentences each. Each 50-set list contains 25 sentences that have high contextual predictability and 25 sentences that have low contextual predictability. That is, the last

Table 3-3. Experimental Conditions

\begin{tabular}{llc}
\hline \multicolumn{1}{c}{$\begin{array}{c}\text { Experimental } \\
\text { Conditions }\end{array}$} & \multicolumn{1}{c}{ Cognitive Skills } & $\begin{array}{c}\text { Listening Condition } \\
\text { SNR }\end{array}$ \\
\hline Simple Dual Task & $\begin{array}{l}\text { Auditory Word Recognition + Visual } \\
\text { Processing } \\
\text { Moderate Dual Task }\end{array}$ & $\begin{array}{l}\text { Auditory Working Memory (isolated } \\
\text { word) + Visual Processing }\end{array}$ \\
Complex Dual Task & $\begin{array}{l}\text { Auditory Working Memory (words } \\
\text { in sentence) + Visual Processing }\end{array}$ & $0,3,5 \mathrm{~dB}$ \\
\hline
\end{tabular}

$\mathrm{SNR}=$ Signal-to-noise ratio 
word of each sentence is either highly predictable from the sentence context or not likely to be predicted from the sentence context. There were a total of three listening conditions, and two of the three experimental conditions (Simple Dual Task and Complex Dual Task) required a complete sentence list. The eight SPIN lists were randomized, and three lists were assigned to each of the two experimental conditions. Thus, a total of six SPIN lists were utilized for each person. The audio-recorded CID W-22 materials (Hirsh, 1952) were used for the Moderate Dual Task. The CID W-22 materials include four recorded lists of 50 words, each. Although the recorded CID W-22 word lists use a carrier phrase before each word, (e.g. "Say the word 'an'."), the carrier phrase was removed so that only the target word was presented (e.g. "Day, Toe, Felt, Stove"). This alteration was made to remove sentence context effects provided by the carrier phrase.

\section{Auditory Task: Procedure}

Each of the dual task conditions were presented at the following signal to noise ratio (SNR) conditions: 5, 3, and $0 \mathrm{~dB}$ SNR, using the multi-talker babble that accompanies the SPIN test as the competing noise. Specifically, the presentation level for the speech was $65 \mathrm{~dB}$ SPL and the noise was varied to effect the above mentioned SNRs. All testing was performed in a double-walled, sound-attenuating booth. The SPIN-R sentences and CID W-22 word lists were presented binaurally through insert earphones EAR-3A (Etymotic Research) routed through a Grason-Stadler 61, two-channel audiometer. The experimental condition (Simple Dual Task, Moderate Dual Task, and Complex Dual Task), listening conditions (0dB SNR, $3 \mathrm{~dB}$ SNR and $5 \mathrm{~dB}$ SNR), SPIN-R sentence list, and CID W-22 word list were randomized for each participant.

\section{Visual Task: Stimuli}

While performing the auditory task, participants also performed a visual task. During the visual task, participants sat in front of a 15-inch computer monitor and were presented with series of letters that appeared in the midfield. The letters consisted of size 40 black font capital letters presented against a white background. The letters were presented for a maximum of 1500 millisecond time limit or until participant response. The screen was erased after each letter stimuli. The stimuli were presented via SuperLab 4.0 stimuli presentation software (Cedrus, 2010). The presentation of the visual task started when the auditory task began and continued until the auditory task was completed. During the Simple Dual Task Condition, the participants were presented with approximately 290 letters and the target letter appeared a minimum of 30 times and a maximum of 40 times. The visual task stimuli presentation varied for Moderate Dual Task and Complex Dual Task conditions in accordance with length of the auditory task presentation. For example, in the simple auditory condition entire lists of 50 sentences were presented to all participants. Whereas for the moderate and complex tasks, the participants received different numbers of items relative to their working memory capacity. Hence, the visual task stimuli presentation varied in length with the number of items administered during the working memory task. 


\section{Visual Task: Procedure}

Within each experimental condition three signal-to-noise ratio conditions were tested. A different target letter (randomly selected) was selected as the target letter for each of the SNR conditions. Participants were instructed to watch the monitor and press the response key as soon as they recognized the pre-determined target letter appear on the screen. The instructions were first presented on paper for participants to read (Appendix A). The experimenter then verbally reinforced the instructions by reading the instructions aloud and did not begin the experiment until the participant indicated that he or she understood the task. The target letter and instructions were presented on the monitor screen before each listening condition. The target letter was randomized for each of the SNR conditions. For each listening condition (0, 3, $5 \mathrm{~dB}$ SNR), a small break was given to reinstruct the participant with the new target letter. Response visual accuracy (correct identification of target letter) for visual processing and visual processing speed (reaction time to letter presentation as measured by the key click) were determined for each trial. Only the processing speed data for accurate responses were analyzed in subsequent analyses. The above describes the general stimuli and procedures used for the Auditory and Visual tasks. The specific experimental condition descriptions follow.

\section{Simple Dual Task Condition}

During the Simple Dual Task condition, participants completed a task of auditory word recognition, while simultaneously performing a visual letter identification task in the presence of noise. The task required participants to repeat the final word heard in each sentence while simultaneously identifying a given letter presented in the visual display. Participants were tested in this dual task in the 3 previously mentioned SNRs. For each listening condition the following measures were obtained: percent correct auditory word recognition, and for visual processing: visual processing speed and percent visual accuracy letter identification.

\section{Moderate Dual Task Condition}

Moderate Dual Task Condition combined a visual processing speed task with a working memory (isolated words) task. Words were presented in list sets of 2, 4, 6, and 8 word list sequences. Within each level of recall $(2,4,6$, or 8$)$, a participant was presented one set of word lists at a time, two sets needed to be completed before the set size was increased to the next set level. Thus, a participant heard one set of two words, and was then was asked to recall the set. If the set was recalled correctly, a second set of two words was introduced. The participant was then asked to recall the set, if the set was correctly recalled, then set size was considered complete. The set size was then increased to a set of four words. Therefore, participants had to successfully remember both sets of words within each level [ie., 1 set of 2 (twice), 1 set of 4 (twice), 1 set of 6 (twice), and 1 set of 8 (twice)]. If a participant missed one of the words in the set, the set was 
considered completed, and the participant was presented with the next set condition. Percent correct for each set was calculated as the total correct divided by the maximum number presented across all participants (32 words) times one hundred. Overall, participants could potentially be presented with a maximum of 40 words if they successfully mastered the 8-item level. Consequently, participants were not all presented with equal number of sentences.

Participants were instructed to listen to the word list and then recall each word heard in the set, preferably in the order they were presented (but any order was accepted). If the participant successfully recalled the words in a set level for two separate sets, then they were presented with the next level in the set hierarchy. An example is given for clarification. In a set of 2 words the participant is presented with the following word list: (1) "been" (2) "house". After both words had been presented, the participant was prompted to recall the two words of the set. The correct answer was "been, house." They were then presented with an additional set of 2 words. If they successfully recalled those, then they moved to the set of 4 words. In a set of 4 words such as (1) "book", (2) "cold", (3) "flew", and (4) "box", the participant was asked to recall each word in the set ("book, cold, flew, box"). If they recalled correctly, they were then presented with an additional set of 4 words. This process was continued for sets of 6 and 8 words. Additionally, participants were asked to simultaneously perform the visual processing task described previously. If a participant missed one of the words in the set, the set was considered completed and participant was presented with the next set condition. This yielded the following measure for working memory — percent correct recall—and for visual processing - percent correct letter identification and processing speed (for accurate visual responses).

\section{Complex Dual Task Condition}

The complex Dual Task Condition combined the visual task with an auditory working memory task for words in sentence context. Although the SPIN sentences were used, they were not used to assess word recognition performance as in the Simple Dual Task condition. Instead, the sentences were presented to assess working memory span for sets of 2, 4, 6, and 8 sentences. Within each level of recall $(2,4,6$, or 8$)$, a participant was presented one set of sentences at a time and asked to recall the final word of each of the sentences in the set. Two sets needed to be completed before the set size was increased to the next set level. Thus, a participant heard one set of two sentences, and was then asked to recall the set. If the set was recalled correctly, a second set of two sentences was introduced. The participant was then asked to recall the set, if the set was correctly recalled, then set size was considered complete. The set size was then increased to a set of four sentences. Hence, participants had to successfully remember both sets of final words in each sentence within each level [ie., 1 set of 2 (twice), 1 set of 4 (twice), 1 set of 6 (twice), and 1 set of 8 (twice)]. Similar to the Moderate Dual Task condition, if a participant missed one of the words in the set, the set was considered completed and participant was presented with the next set condition. Scoring for each set was calculated using the total correct divided by the maximum number presented across all participants 
(32 words) times one hundred. In total, participants could be presented with a maximum of 40 sentences if they successfully mastered the 8-item level. As noted in earlier conditions, participants were not all presented with equal number of sentences.

During the Complex Dual Task Condition, as in the previous perception task, the participant was not allowed to repeat the last word immediately after he or she heard it. Instead, the participant was instructed to listen to the sentence, to remember the last word of each of the sentences, and to recall the last word of each of the sentences after all sentences in the set had been presented. Once a set was presented, the participant was prompted to recall the last word of each of the sentences heard in the set, preferably in the order they were presented (but any order was accepted). If the participant successfully recalled the words in a set level for two separate sets, then they were presented with the next level in the set hierarchy. An example is provided for clarity. In a set of 2 sentences the participant is presented with the following sentences: (1) "The sky is blue." (2) "The grass is green." The participant was then prompted to recall the last words of the two sentences after both sentences had been presented. The correct answer was "blue" and "green". The participant was then presented with an additional set of 2 sentences. If they successfully recalled those, then they moved to the set of 4 sentences. In a set of 4 sentences such as (1) "The door is broken." (2) "The banana is old." (3) "The yellow car is new." and (4) "He walked the dog." The participant was asked to recall the last word of each sentence ("broken, old, new, dog"). If they recalled correctly, they were then presented with an additional set of 4 words. This process was continued for sets of 6 and 8 words. Additionally, participants were asked to simultaneously perform the visual processing task described previously. Prior to the beginning of the Complex Dual task, participants were given written and verbal instructions for both the Visual processing task and the word recall task. To summarize, in this experimental condition participants were engaged in the visual task as they listened to the sets of sentences and were asked to recall the final words in the sets. Thus, participants simultaneously responded to the visual stimuli by clicking the response pad and the auditory stimuli by verbally recalling words. This yielded the following measures for working memory: percent correct recall. Percent correct recall was calculated as follows: the total correct divided by the maximum number presented across all participants (32 words) times one hundred. For visual processing, the following measures were obtained: percent correct for visual letter identification and processing speed (for accurate visual responses). 


\section{CHAPTER 4. RESULTS}

The study was a between-group $3 \times 3 \times 2$ design with group, noise, and task as independent variables, and cognitive performance as the dependent variable. Statistical analyses were conducted for auditory or visual accuracy and processing speed measures across task, noise, and group condition. A Doubly Repeated Mixed Multivariate Analysis, including Multivariate, Univariate, and Pairwise Comparisons, was conducted. All percent accuracy scores were transformed using arcsine transformation prior to analysis. Group data across dual task conditions is shown in Appendix B. Overall raw group data is included for reference, see Table 4-1. Group data for Simple Dual Task Condition is included in Figures B-1, B-2, and B-3. Group data for Moderate Dual Task Condition is in Figures B-4-, B-5, and B-6. Finally, group data for Complex Dual Task Condition is located in Figures B-7, B-8, and B-9.

\section{Doubly Repeated Mixed Multivariate Analysis}

A 3 X 3 X 2 Doubly Repeated Mixed Multivariate ANOVA was performed. Task Complexity (Simple, Moderate, Complex), Listening Condition (0 dB SNR, 3 dB SNR, 5 dB SNR), and Group (Group1: younger, Group 2: older) were factors, with auditory accuracy, visual accuracy, and visual processing speed as dependent variables. Results indicated one significant omnibus effect. Specifically, there is a significant effect of Task [Wilks' Lambda $\mathrm{F}(6,100)=103.09, \mathrm{p}=<.001, \eta 2=.856$ ]. The Task by Noise interaction approached significance [Wilks' Lambda $\mathrm{F}(12,280.74)=1.66, \mathrm{p}=.077, \eta 2=.058]$. There was no significant omnibus effect of Noise, Group, Task*Group, Task*Noise, Noise*Group, or Task*Noise*Group interaction.

\section{Task Analysis}

The univariate analysis within the larger 3 X 3 X 2 Doubly Multivariate Repeated Measures ANOVA yielded examination of the effect of one factor (e.g. Task) across all levels of one other factor (e.g. Listening condition at $0 \mathrm{~dB}$ SNR, $3 \mathrm{~dB}$ SNR, $5 \mathrm{~dB}$ SNR), ,and indicated a significant main effect of Task on auditory accuracy measures $[\mathrm{F}(1.75$, 47.28) $=990.94, \mathrm{p}=<.001 \eta 2=.973$, (Greenhouse-Geisser Adjustment)], visual accuracy $[\mathrm{F}(1.87,50.46)=15.17, \mathrm{p}=<.001, \eta 2=.360$, (Greenhouse-Geisser Adjustment)], and visual processing speed $[\mathrm{F}(1.86,50.14)=18.87, \mathrm{p}=<.001, \eta 2=.411$, (Greenhouse-Geisser Adjustment)]. The within subject tests for auditory accuracy, visual accuracy, and reaction time will be explored below.

\section{Task Analysis: Auditory Accuracy}

A Test of Within Subjects Contrasts was conducted to further explore the main effect for Task. Task was significant for Auditory Accuracy, with differences between 
Table 4-1. Dual Task Auditory, Visual Accuracy, and Speed Average Scores

\begin{tabular}{|c|c|c|c|c|c|c|}
\hline \multirow{2}{*}{$\begin{array}{l}\text { Listening } \\
\text { Condition } \\
\end{array}$} & \multicolumn{2}{|c|}{ Auditory Accuracy (\%) } & \multicolumn{2}{|c|}{ Visual Accuracy (\%) } & \multicolumn{2}{|c|}{ Speed (ms) } \\
\hline & Group 1 & Group 2 & Group 1 & Group 2 & Group 1 & Group 2 \\
\hline $5 \mathrm{~dB}$ & $\begin{array}{l}96.80 \% \\
(\mathrm{SD}=.03)\end{array}$ & $\begin{array}{l}93.87 \% \\
(\mathrm{SD}=.04)\end{array}$ & $\begin{array}{l}\text { SIMPLE } \\
93 \% \\
(\mathrm{SD}=.22)\end{array}$ & $\begin{array}{l}99 \% \\
(\mathrm{SD}=.02)\end{array}$ & $\begin{array}{l}610.09 \\
(\mathrm{SD}=229.85)\end{array}$ & $\begin{array}{l}593.92 \\
(\mathrm{SD}=95.69)\end{array}$ \\
\hline $3 \mathrm{~dB}$ & $\begin{array}{l}94.27 \% \\
(\mathrm{SD}=.05)\end{array}$ & $\begin{array}{l}91.20 \% \\
(\mathrm{SD}=.06)\end{array}$ & $\begin{array}{l}99 \% \\
(\mathrm{SD}=.02)\end{array}$ & $\begin{array}{l}97 \% \\
(\mathrm{SD}=.06)\end{array}$ & $\begin{array}{l}559.71 \\
(\mathrm{SD}=88.97)\end{array}$ & $\begin{array}{l}6176.18 \\
(\mathrm{SD}=104.63)\end{array}$ \\
\hline \multirow[t]{2}{*}{$0 \mathrm{~dB}$} & $\begin{array}{l}92.13 \% \\
(\mathrm{SD}=.05)\end{array}$ & $\begin{array}{l}88.67 \% \\
(\mathrm{SD}=.06)\end{array}$ & $\begin{array}{l}98 \% \\
(\mathrm{SD}=.03)\end{array}$ & $\begin{array}{l}98 \% \\
(\mathrm{SD}=.04)\end{array}$ & $\begin{array}{l}565.83 \\
(\mathrm{SD}=95.14)\end{array}$ & $\begin{array}{l}661.53 \\
(\mathrm{SD}=216.69)\end{array}$ \\
\hline & & & \multicolumn{2}{|c|}{ MODERATE } & & \\
\hline $5 \mathrm{~dB}$ & $\begin{array}{l}19.42 \% \\
(\mathrm{SD}=24.58)\end{array}$ & $\begin{array}{l}16.46 \% \\
(S D=20.07)\end{array}$ & $\begin{array}{l}85 \% \\
(\mathrm{SD}=.21)\end{array}$ & $\begin{array}{l}94 \% \\
(\mathrm{SD}=.10)\end{array}$ & $\begin{array}{l}787.39 \\
(\mathrm{SD}=223.38)\end{array}$ & $\begin{array}{l}721.80 \\
(\mathrm{SD}=130.78)\end{array}$ \\
\hline $3 \mathrm{~dB}$ & $\begin{array}{l}8.26 \% \\
(S D=5.96)\end{array}$ & $\begin{array}{l}16.30 \% \\
(S D=12.51)\end{array}$ & $\begin{array}{l}90 \% \\
(\mathrm{SD}=.18)\end{array}$ & $\begin{array}{l}91 \% \\
(\mathrm{SD}=.18)\end{array}$ & $\begin{array}{l}726.39 \\
(\mathrm{SD}=173.52)\end{array}$ & $\begin{array}{l}788.26 \\
(S D=270.48)\end{array}$ \\
\hline $0 \mathrm{~dB}$ & $\begin{array}{l}18.97 \% \\
(\mathrm{SD}=.15)\end{array}$ & $\begin{array}{l}10.21 \% \\
(\mathrm{SD}=.9 .02)\end{array}$ & $\begin{array}{l}88 \% \\
(\mathrm{SD}=.22)\end{array}$ & $\begin{array}{l}75 \% \\
(\mathrm{SD}=.38)\end{array}$ & $\begin{array}{l}725.64 \\
(\mathrm{SD}=281.106)\end{array}$ & $\begin{array}{l}839.98 \\
(\mathrm{SD}=348.45)\end{array}$ \\
\hline $5 \mathrm{~dB}$ & $\begin{array}{l}29.46 \% \\
(\mathrm{SD}=.20 .61)\end{array}$ & $\begin{array}{l}21.04 \% \\
(\mathrm{SD}=7.86)\end{array}$ & $\begin{array}{l}\text { COMPLE) } \\
79.89 \% \\
(\mathrm{SD}=.35)\end{array}$ & $\begin{array}{l}77.69 \% \\
(\mathrm{SD}=.32)\end{array}$ & $\begin{array}{l}829.85 \\
(\mathrm{SD}=322.92)\end{array}$ & $\begin{array}{l}786.37 \\
(\mathrm{SD}=249.73)\end{array}$ \\
\hline $3 \mathrm{~dB}$ & $\begin{array}{l}24.33 \% \\
(\mathrm{SD}=11.70)\end{array}$ & $\begin{array}{l}21.88 \% \\
(\mathrm{SD}=11.88)\end{array}$ & $\begin{array}{l}94.52 \% \\
(\mathrm{SD}=.08)\end{array}$ & $\begin{array}{l}80.03 \% \\
(\mathrm{SD}=.27)\end{array}$ & $\begin{array}{l}666.85 \\
(S D=132.65)\end{array}$ & $\begin{array}{l}799.83 \\
(\mathrm{SD}=232.93)\end{array}$ \\
\hline $0 \mathrm{~dB}$ & $\begin{array}{l}29.02 \% \\
(\mathrm{SD}=23.75)\end{array}$ & $\begin{array}{l}20.83 \% \\
(\mathrm{SD}=12.83)\end{array}$ & $\begin{array}{l}88.38 \% \\
(\mathrm{SD}=.096)\end{array}$ & $\begin{array}{l}80.30 \% \\
(\mathrm{SD}=.25)\end{array}$ & $\begin{array}{l}698.88 \\
(\mathrm{SD}=147.828)\end{array}$ & $\begin{array}{l}763.91 \\
(\mathrm{SD}=250.97)\end{array}$ \\
\hline
\end{tabular}


Simple DT and Moderate DT $[\mathrm{F}(1,27)=1172.11, \mathrm{p}=<.001, \eta 2=.977]$ and Simple DT and Complex DT $[\mathrm{F}(1,27)=1617.79, \mathrm{p}=<.001, \eta 2=.984]$. Additional exploration of the main effects for Task were conducted using Pairwise Comparisons with Bonferroni corrections. It was found that all of the pairwise comparisons for Auditory Accuracy were statistically significant $(\mathrm{p}<.001)$. These differences suggest that for the Moderate DT $(\mathrm{M}=.153)$ participants were less accurate than at the Simple DT $(\mathrm{M}=1.23)$ and the Complex DT $(\mathrm{M}=.247)$ levels. The results also suggest that the moderate task is significantly different (poorer) than the simple task and the complex task. See Table 4-2 and Figure 4-1 for Auditory Accuracy Pairwise Comparison details.

\section{Task Analysis: Visual Accuracy}

The within subjects contrast for Task was significant for Visual Accuracy with differences between Simple DT and Moderate DT $[\mathrm{F}(1,27)=9.778, \mathrm{p}=.004, \eta 2=.266]$, and Simple DT and Complex DT $[\mathrm{F}(1,27)=29.77, \mathrm{p}=<.001, \eta 2=.524]$. Using Pairwise Comparisons with Bonferroni corrections, it was found that the pairwise comparisons for Visual Accuracy were statistically significant $(\mathrm{p}<.05)$. Results suggest that in the Simple DT, participants were more accurate $(\mathrm{M}=1.45)$ than at the Moderate DT $(\mathrm{M}=1.26)$ and the Complex DT $(M=1.16)$ levels. These findings indicate that Simple DT was significantly different from both Moderate DT and Complex DT. Unlike the Task Analysis: Auditory Accuracy findings, Moderate DT is not significantly different from the Complex DT as hypothesized. See Table 4-2 and Figure 4-2 for Auditory Pairwise Comparison details.

\section{Task Analysis: Reaction Time}

Finally, the within subject contrast revealed that Task was significant for Visual Processing Speed as measured by reaction time with differences between Simple DT and Moderate DT $[\mathrm{F}(1,27)=23.34, \mathrm{p}=<.001, \eta 2=.464]$ and Simple DT and Complex DT $[F(1,27)=33.27, p=<.001, \eta 2=.553]$. Pairwise Comparisons with Bonferroni corrections indicated that the Visual Processing Speed Reaction Time was statistically significant $(p<.001)$. Specifically, the processing speed for the simple task was significantly different than for the moderate and the complex tasks. However, the visual processing speed for the moderate task was not significantly different from the complex task. These differences suggest that for the Simple DT participants were faster $(M=600.61)$ than at the Moderate DT $(M=765.32)$ and the Complex DT $(M=757.61)$ levels. See Table 4-2 and Figure 4-3 for Auditory Pairwise Comparison details. 
Table 4-2. Comparison of Overall Task Performance of 95\% Confidence Intervals

\begin{tabular}{|c|c|c|c|c|c|}
\hline Measure & Task & Mean & SE & P-value & $\begin{array}{c}\mathbf{9 5 \%} \text { Confidence } \\
\text { Interval } \\
\end{array}$ \\
\hline \multicolumn{6}{|c|}{ Pairwise Means } \\
\hline \multirow[t]{3}{*}{ Auditory Accuracy } & Simple Dual Task Condition & 1.24 & .024 & NA & {$[1.185,1.284]$} \\
\hline & Moderate Dual Task Condition & .153 & .024 & NA & {$[.104, .203]$} \\
\hline & Complex Dual Task Condition & .247 & .021 & NA & {$[.203, .292]$} \\
\hline \multirow[t]{3}{*}{ Visual Accuracy } & Simple Dual Task Condition & 1.45 & .033 & NA & {$[1.378,1.512]$} \\
\hline & Moderate Dual Task Condition & 1.26 & .065 & NA & {$[1.127,1.395]$} \\
\hline & Complex Dual Task Condition & 1.16 & .057 & NA & {$[1.039,1.275]$} \\
\hline \multirow[t]{3}{*}{ Processing Speed } & Simple Dual Task Condition & 600.61 & 23.00 & NA & {$[553.414,647.802]$} \\
\hline & Moderate Dual Task Condition & 765.32 & 37.87 & NA & {$[687.613,843.033]$} \\
\hline & Complex Dual Task Condition & 757.61 & 33.62 & NA & {$[688.629,826.597]$} \\
\hline \multicolumn{6}{|c|}{ Pairwise Mean Differences } \\
\hline \multirow[t]{3}{*}{ Auditory Accuracy } & Simple vs. Moderate & $1.082 *$ & .032 & .000 & {$[1.001,1.162]$} \\
\hline & Simple vs. Complex & $.987^{*}$ & .025 & .000 & {$[.925,1.050]$} \\
\hline & Moderate vs. Complex & $-.094 *$ & .024 & .002 & {$[-.155,-.033]$} \\
\hline \multirow[t]{3}{*}{ Visual Accuracy } & Simple vs. Moderate & $.184^{*}$ & .059 & .013 & {$[.034, .334]$} \\
\hline & Simple vs. Complex & $.288^{*}$ & .053 & .000 & {$[.153, .423]$} \\
\hline & Moderate vs. Complex & .104 & .047 & .101 & {$[-.015, .223]$} \\
\hline \multirow[t]{3}{*}{ Processing Speed } & Simple vs. Moderate & $-164.715^{*}$ & 34.093 & .000 & {$[553.414,647.802]$} \\
\hline & Simple vs. Complex & $-157.005^{*}$ & 27.180 & .000 & {$[687.613,843.033]$} \\
\hline & Moderate vs. Complex & 7.710 & 29.102 & 1.000 & {$[688.629,826.597]$} \\
\hline
\end{tabular}

*significant at the .05 level 


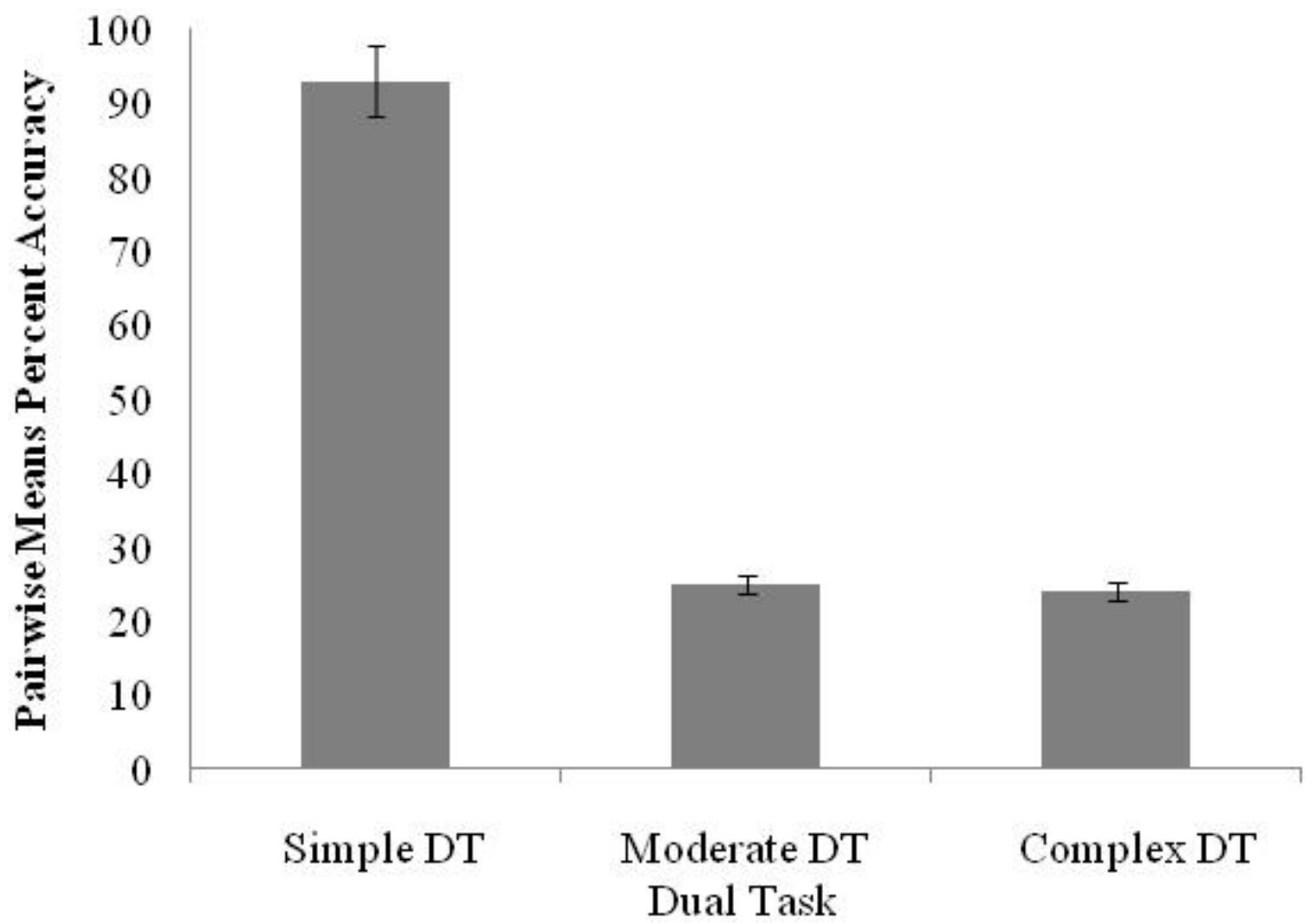

Figure 4-1. Pairwise Means in Auditory Accuracy Task Performance 


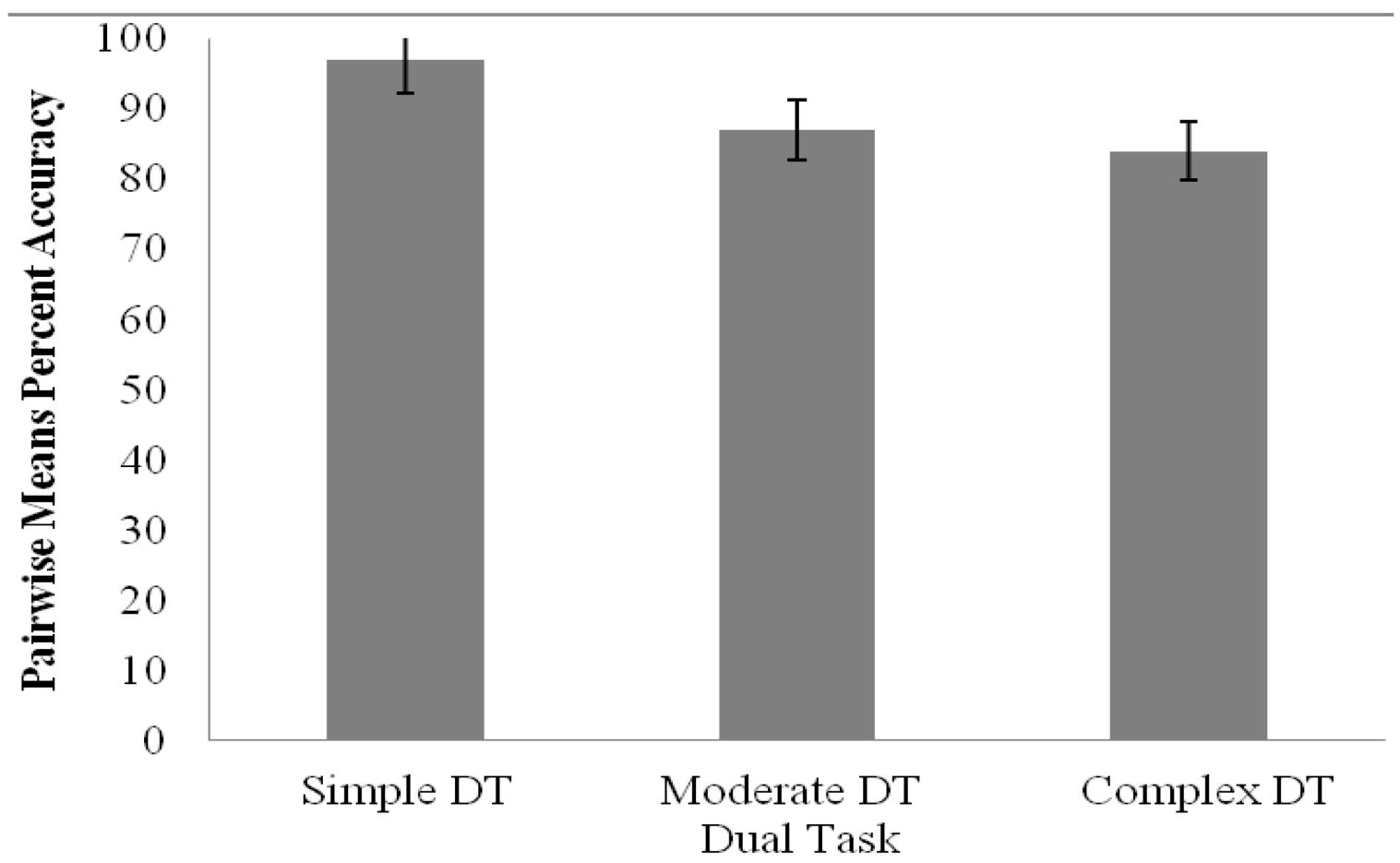

Figure 4-2. Pairwise Means in Visual Accuracy Task Performance 


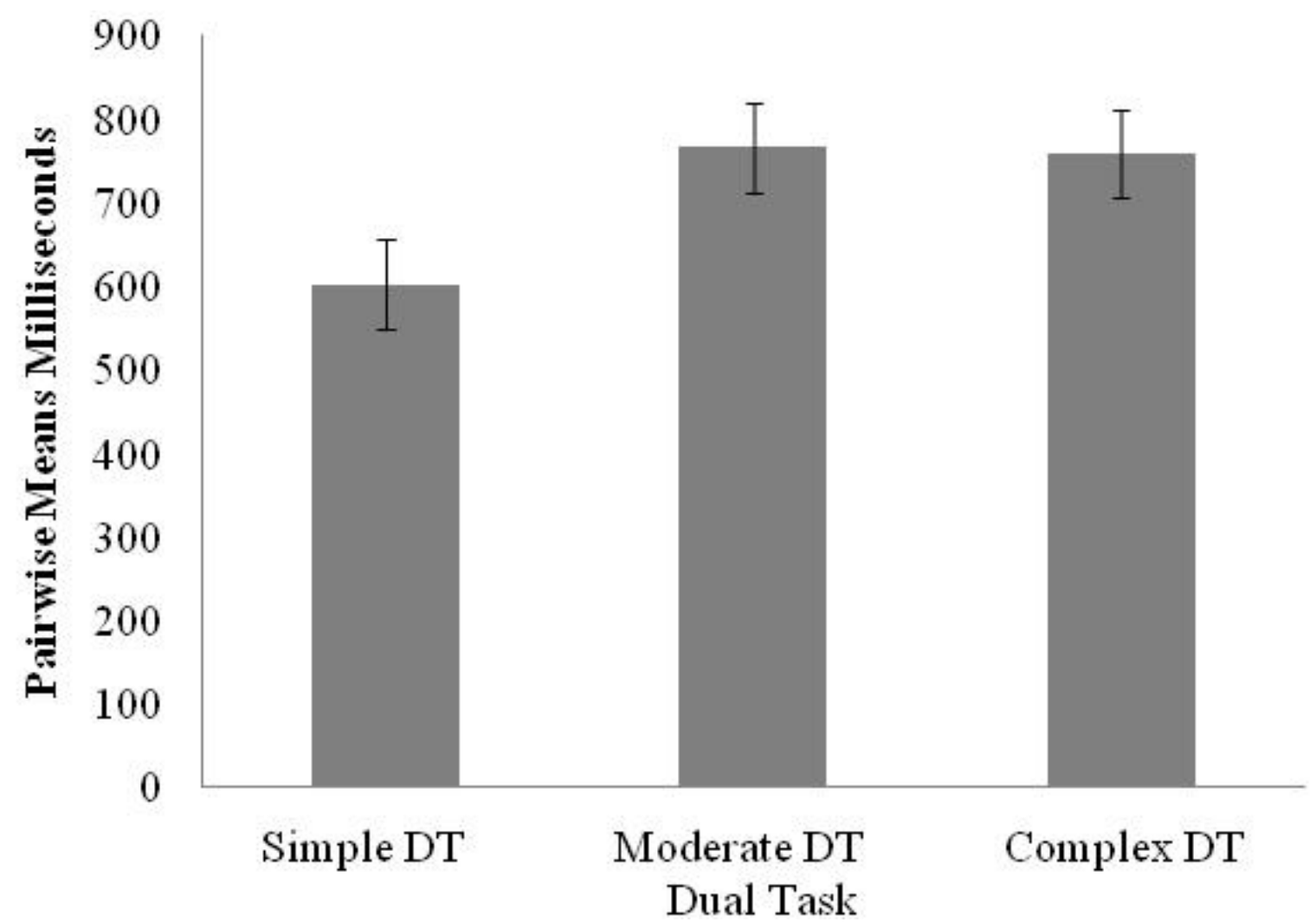

Figure 4-3. Pairwise Means in Processing Speed Task Performance 


\section{CHAPTER 5. DISCUSSION}

The current study examined the effects of noise on cognitive function during dual task conditions of varying complexity across groups. Research has shown that structural complexity of a stimuli (i.e. phonologically complex stimuli such as words versus nonsense words) can affect auditory or visual accuracy and reaction time measures (Carroll, 2012). The goal of this study was to systematically increase cognitive load and test the effects of different levels of noise on auditory or visual accuracy and processing speed measures in young and old adults. The present study focused on a Simple dual task [visual processing + word recognition], Moderate dual task [visual processing + working memory (word)], and Complex dual task [visual processing + working memory (sentence)] conditions in noise. The hypotheses predicted: (1) participants for both younger and older groups would exhibit performance decline (with the older group demonstrating a more significant decline) of cognitive skill with increased task complexity and/or increased difficulty in SNR; (2) with each progressive increase in cognitive load (task), participants would demonstrate decreased auditory or visual accuracy and slower reaction times; and (3) with each progressive increase in perceptual load (noise), participants would demonstrate decreased auditory or visual accuracy and slower reaction times.

Hypothesis one proposes that participants in both younger and older groups would exhibit performance decline (with the older group demonstrating a more significant decline of cognitive skill) with increased task complexity and/or increased difficulty in SNR. This hypothesis was not supported by the data. There were no group differences observed outside of the Group * Noise interaction discussed above. These data indicate that although there were significant effects for task and noise independently, both groups performed similarly. In other words, as task complexity became more challenging, both groups were able to produce similar performance scores. This lack of group differences may have several possible implications. First, this finding indicates that the Perceptual and Cognitive Load Model may equally apply to both older and younger individuals. Second, the lack of difference between groups may be evidence for the decline compensation theory which suggests that older individuals process information using more brain areas than younger adults to produce the same results. Literature has shown that older and younger individuals demonstrate differing brain activation patterns (Cabeza et al., 2002; Davis et al., 2008; Getzman, 2011; Peelle et al., 2010). Finally, the age ranges for each group may have been a confounding factor. There may not have been a substantial enough difference in age of the groups, leading to similar group performances.

Hypothesis two proposed that with each progressive increase in cognitive load (task), participants would demonstrate decreased auditory or visual accuracy and slower reaction time. The Doubly Multivariate Repeated Measures ANOVA yielded omnibus and main effects that do support this hypothesis. These findings indicate a general significance of Task when considering all other factors. The results suggest that Task complexity has a significant effect on cognitive function. Furthermore, it indicates that 
this effect is present across dependent variables of auditory accuracy, visual accuracy, and visual processing speed. In terms of visual processing, auditory and visual accuracy decreased and reaction time increased with increased cognitive load. This was the predicted pattern. Overall, these results support the hypothesis that decreased auditory or visual accuracy and slower reaction time would be observed during tasks of increased complexity during visual processing.

Auditory accuracy results revealed that Moderate Dual Task was significantly different than Simple and Complex Dual Tasks across auditory accuracy measures. The Moderate Dual Task showed poorer auditory accuracy than the Simple and Complex Dual Tasks. This did not follow a predictable pattern. These results suggest that the Moderate DT is more challenging than the Complex DT, for auditory accuracy. In designing the difficulty level of the tasks, it was predicted that words in isolation would be less tasking than words presented in sentence context. However, the results from the present study suggest that words in isolation maybe more challenging than words in a sentence. It was predicted that the recall of words in a sentence would be more challenging because additional cognitive processing would be required to process the syntactic and semantic information in the sentence. However, the recall of words in isolation may be more challenging due to lack of context of surrounding words. Thus, sentence context makes the task easier. Further, the mid-condition of the Moderate Task was introduced in this study to examine significant findings of the Harvey et al. study which suggested that changes in cognitive function maybe occurring between a word recognition dual task and a working memory for words in sentences dual task. Therefore, the $2 \mathrm{~b}$ sub hypotheses predicted that there would be a significant performance difference at the mid-level condition. This was evident for auditory accuracy, where the Moderate Task is more challenging than the Simple or Complex Dual Tasks.

Hypothesis two also suggests that with each progressive increase in perceptual load (noise), participants would demonstrate decreased auditory or visual accuracy and slower reaction times. This hypothesis was not supported by the data. Consequently, the 2a sub hypothesis was also not supported.

\section{Perceptual and Cognitive Load Model}

The current study findings support the Mattys et al. (2009) Perceptual and Cognitive Load Model of energetic and informational masking. This model asserts that cognitive load is composed of perceptual and cognitive loads: "Perceptual load is any alteration to the signal leading to diminish acoustic integrity." (Mattys et al., 2009, p. 2). Cognitive load is described as any load that "arises from the recruitment of central processing resources due to concurrent attentional or mneumonic processing" (Mattys et al., 2009, p. 2). They further stipulate that perceptual and cognitive load can be differentiated using energetic and informational masking. These results are consistent with this Model, which suggests that as cognitive skill changes energetic masking transitions to informational masking as higher level complexity of tasks change. Future 
research should contribute to the growing body of experimental literature and add to the support of unification of cognition and noise theory.

These results indicate that performance break down (in common difficult noise conditions) occurs during more complex dual tasks. This is consistent with Mattys et al. (2009) assertion that noise can have differing effects on speech intelligibility and cognitive skills. It can affect speech intelligibility in simple tasks while only affecting working memory in more complex tasks. These results suggest that as cognitive skill changes, energetic masking transitions to informational masking as the higher level complexity of tasks change. Therefore, although the Perceptual and Cognitive load model does not directly address changes according to age, our findings support the argument made by researchers that the extent of the effect of energetic masking is related to both the level of noise and the complexity of the task itself. Hence, an energetic masker can become an informational masker, simply by changing the complexity of task. This has implications for aging individuals. Due to cognitive function changes over time, it can be hypothesized that as individuals age maskers that provide energetic masking at a young age will result in informational masking for older individuals. Further research and analyses are necessary to determine the extent to which each noise level affects cognitive function at each level of cognitive load across age ranges. Alternatively, the decline compensation hypothesis and Pichora-Fuller et al. (1995) assert that the alterations in performance are due to modifications in auditory processing and neurophysiological functioning due to age, respectively. The alternative justifications for cognitive changes may align directly with the perception of the cognitive load model. Further research his warranted to examine the role of age in cognitive wellness and speech in noise research.

Finally, a Group by Noise interaction was also observed. Group 1 (younger) consistently performed faster than Group 2 (older) for 0 and $3 \mathrm{~dB}$ SNR conditions; however, at $5 \mathrm{~dB}$ SNR, older individuals performed faster than younger participants. The performance of older individuals at 5dB SNR was unexpected; rather, it was hypothesized that there would be an alteration in function at $3 \mathrm{~dB}$ SNR. Overall, these results support the Hypothesis two, that participants would demonstrate decreased auditory or visual accuracy and slower reaction times during increasingly difficult SNR conditions. This finding confirms common observation that noise in the environment can affect cognitive function. The change in performance at $5 \mathrm{~dB}$ SNR for the older group might be attributed to a significant increase in cognitive resources when tasks are performed in the easiest listening condition. This assertion would support that younger individuals use fewer cognitive resources than older individuals when completing a task, as noted by the decline compensation hypothesis. Thus, older individuals will experience a more significant increase in allocated resources because of the greater demand due to dual task in noise. The Noise by Group interaction supports similar findings to PichoraFuller et al. (1995) for alteration in performance in older and younger individuals during different listening conditions. Pichora-Fuller et al. found that younger listeners achieve maximal advantage during more challenging SNRs than older listeners. For example, the older group demonstrated more benefit from quiet listening conditions than +8 and $+5 \mathrm{~dB}$ SNR when compared to younger participants. This is similar to the current finding that older individuals performed quicker than younger participants in the easiest listening 
condition $+5 \mathrm{~dB}$ SNR. These findings indicate listening conditions can differentially alter cognitive function across age groups, particularly for processing speed.

The results of the auditory and visual accuracy and processing speed analyses indicate that auditory or visual accuracy and visual processing speed are affected by task complexity. This finding expands upon the previous results (Harvey et al., in preparation; Pichora-Fuller, 1995) where researchers found that auditory accuracy alone was affected by noise and task complexity. The current study added systematic examination of dual task, which allowed for direct within-group comparison between the simple dual task, moderate dual task, and complex dual task. Further, these results parallel findings by Sarampalis et al. (2009) made that auditory noise affects cognitive performance for dual tasks involving working memory and processing speed. Sarampalis et al. (2009) tested dual tasks including: (1) two auditory tasks and (2) an auditory and processing speed dual task. Although the latter dual task used is similar to the Dual Task and Complex Dual Task utilized in this study, the cognitive skills varied and were not examined systematically. These results suggest that the type of task contributes to concurrent cost in task performance.

\section{Cognitive Load Levels}

The investigation of dual task is important for the study of listening effort across age groups and cognitive skill (Gosselin, 2010; Holtzer, 2005). Previous literature did not compare accuracy and speed performance for tasks in isolation and dual task. The current study findings indicate that processing speed is affected by cognitive load. Additionally, it is suggested that various combinations of complex dual tasks may be significantly affected by noise and cognitive loading depending upon the type of activity. Research has scarcely examined the effects of cognitive load with various levels of noise across age groups. Early literature has primarily focused on establishing performance in younger groups; however, the primary target population is the older population, those having difficulty with cognitive skill sets, noise, Research examining these cognitive load variables should focus on older individuals. Furthermore, the cognitive skills examined should represent frequently used cognitive skills for tasks of everyday living. Even more precisely, those tasks employing working memory and processing speed (which are known to decline naturally with age), should be utilized in speech in noise research. Future research is needed to refine and further investigate various combinations of higher level complex dual tasks comprised of frequently utilized cognitive skill under noise levels equal to those of activities of daily living and across age groups.

The current study expanded upon these cognitive load variables by increasing dual task complexity, including mid-condition noise levels, and refining participant criteria to include two groups. The findings suggested that individuals presented with tasks of high complexity or $0 \mathrm{~dB}$ SNR and $3 \mathrm{~dB}$ SNR of noise or greater will demonstrate significant decline in performance compared to less challenging task conditions. For instance, hard tasks and tasks with a lot of noise are significantly more challenging compared to similar tasks of easier complexity or noise conditions. These findings 
support previous literature and contribute to the understanding of cognitive function across noise conditions. Furthermore, these findings support the perceptual and cognitive load theory that both noise and task complexity have a differential effect on cognitive function output. It is suggested that cognitive theories including energetic and informational masking together account for cognitive performance outcomes across task complexities. Based upon these findings, cognitive processing breakdown occurs between Simple dual task and Moderate and/or Complex dual task difficulty and between $5 \mathrm{~dB}$ SNR and noise levels at $0 \mathrm{~dB}$ SNR and $3 \mathrm{~dB}$ SNR.

\section{Limitations}

The current study modified and refined methodology in speech-in-noise research as applied to cognitive load. However, there were a number of areas for improvement in future research. First, the participant sample size may impact results if larger sample size was obtained. Due to the continued change of cognition and neurocognitive processes with age, there is no clear way to separate age groups. However, including a ten year age difference between groups may yield a more accurate representation of the differences in age groups. Future research may also present with obstacles for sample size because populations with disorders are less frequent and therefore have smaller sample sizes. Second, visual accuracy and processing speed stimuli utilizing the Superlab software may be modified to present a more complex visual stimuli or visual response. Participants consistently came close to or ceilinged out of the visual task. This performance suggests that a more challenging visual task maybe warranted. Third, the CID W-22 stimuli were manually modified to exclude the leading phrase (i.e. "number one"). Further review of the stimuli or use of alternative standardized stimuli could better ensure stimuli validity/standardization. Fourth, the dual task hierarchy warrants review. The current study included recall of words in isolation part of the Moderate DT with recall of words in a sentence part of the Complex DT. The reasoning here suggested that recall of words in a sentence would be harder because of the additional cognitive processing for the remainder of the syntactic and semantic information due to additional words. However, the recall of words in isolation may be more challenging due to lack of context of surrounding words. The current protocol introduces possible modifications to the study of dual task in noise. From this protocol further refinement is necessary to ensure systematic examination of dual task, while accounting for dual task validity.

\section{Future Research}

\section{Implications of Results}

These results add to the growing body of literature examining the link between noise and cognitive function. Investigation of noise in normally-aging individuals 40-59 and $60+$ years old targets the population experiencing hearing decline with age. 


\section{Future Questions}

The long term goal of this study is to understand the auditory and cognitive processes that affect cognitive function output across cognitive levels, auditory skill, and age group.

- Is there a group difference between individuals with normal cognitive aging and those with non-normal cognition between dual tasks on cognitive load under different listening conditions?

- Is there a group difference between individuals with normal cognitive aging and those with non-normal cognition in combinations of complex cognitive functions, such as reaction time and working memory, under different listening conditions?

- Is there a group difference between individuals with normal hearing and those with non-normal hearing between dual tasks on cognitive load under different listening conditions?

- Is there a group difference between individuals with normal hearing and those with non-normal hearing in combinations of complex cognitive functions such as reaction time and working memory under different listening conditions? 


\section{LIST OF REFERENCES}

Anderson, S., Parbery-Clark, A., Yi, H., \& Kraus, N. (2011). A neural basis of speechin-noise perception in older adults. Ear and Hearing, 32(6), 750-757.

Austin, D. F., Konrad-Martin, D., Griest, S., McMillan, G. P., McDermott, D., \& Fausti, S. (2009). Diabetes-related changes in hearing. Laryngoscope, 119, 1788-1796.

Baddeley, A. D., Kopelman, M. D., \& Wilson, B. A. (2002). The Handbook of Memory Disorders (2nd ed.). West Sussex, England: John Wiley and Sons.

Bilger, R. C., Nuetzel, J. M., Rabinowitz, W. M., \& Rzeczkowski, C. (1984). Standardization of a test of speech perception in noise. Journal of Speech Hearing Research, 27(1), 32-48.

Birren, J.E., \& Schaie, K.W. (1996). Handbook of Psychology of Aging, $4^{\text {th }}$ ed. San Diego, CA: Academic Press.

Brant, L. J. \& Fozard, J.L. (1990). Age changes in pure-tone hearing thresholds in a longitudinal study of normal human aging. Journal of the Acoustical Society of America, 88(2), 813-820.

Cabeza, R., Anderson, N., Locantore, J.K., \& McIntosh, A.R. (2002). Aging gracefully: Compensatory brain activity in high-performing older adults. NeuroImage, 17, 1394-1402.

Carroll, R., \& Ruigendijk, E. (2012). The effects of syntactic complexity on processing sentences in noise. Journal of Psycholinguist Research, in press. Epub ahead of print retrieved January 15, 2013, from http://link.springer.com/article/10.1007\%2Fs10936-012-9213-7.

Cedrus Corporation. (2008). SuperLab (Version 4.5) [Computer Software]. United States.

Cheng, Y. J., Gregg, E. W., Saaddine, J. B., Imperatore, G., Zhang, X., \& Albright, A. L. (2009). Three decade change in the prevalence of hearing impairment and its association with diabetes in the United States. Preventative Medicine, 49, 360364.

Daneman, M. \& Carpenter, P.A. (1980). Individual differences in working memory and reading. Journal of Verbal Learning and Verbal Behavior, 19, 450-466.

Davis, S.W., Dennis, N.A., Fleck, M., Daselaar, S.M., \& Cabeza, R. (2008). Que PASA?: the posterior-anterior shift in aging. Cerebral Cortex, 18(5), 1201-1209. 
Duncan, S. \& Feldman Barrett, L. (2007). Affect is a form of cognition: A neurobilogical analysis. Cognition and Emotion, 21(6), 1184-1211.

Gates, G.A., (2009). Central auditory processing in presbycusis: an epidemiologic perspective. International adult conference: The Challenge of Aging: Chapter 4. Cover Hearing Care for Adults - the challenge for aging (pp.47-52). Chicago, IL.

Gates, G. \& Mills, J.H. (2005). Presbycusis. The Lancet, 366 (9491), 1111-1120.

Gates G. A., Cobb J. L., D’Agostino R. B.,\& Wolf, P. A. (1993). The relation of hearing in the elderly to the presence of cardiovascular disease and cardiovascular risk factors. Archives of Otolaryngology-Head \& Surgery, 119, 156-161.

Gates, G., \& Cooper, J.C. (1991). Incidence of hearing decline in the elderly. Acta OtoLaryngologica, 111, 240-248.

Getzmann, S. (2012). Handicapped due to age? Behavioral and electrophysiological correlates of speech perception of dichotically presented narratives in young and middle-aged listeners. Journal of Psychophysiology, 26(3), 132-144.

Getzmann, S., \& Falkenstein, M. (2011). Understanding of spoken language under challenging listening conditions in younger and older listeners: A combined behavioral and electrophysiological study. Brain Research, 14, 8-22.

Giovanello, S., \& Verfaellie, M. (2001). The relationship between recall and recognition in amnesia: Effects of matching recognition between patients with amnesia and controls. Neuropsychology, 15(4), 444-451.

Gosselin, P., \& Gagne, J. P. (2010). Use of dual-task paradigm to measure listening effort. Canadian Journal of Speech Language Pathology and Audiology, 34(1), 43-51.

Haga, S., Shinoda, H., \& Kokubun, M. (2002). Effect of task difficulty and time-on-task on mental workload. Japanese Psychological Research, 44, 134-143.

Hargie, O. (2006). The Handbook of Communication Skills, $3^{\text {rd }}$ ed. East Sussex, England: Routledge.

Harvey, J., King, K.A., von Hapsburg, D. (in preparation). Effects of noise on increasing cognitive load in normally aging adults. Target Journal: Journal of Speech, Language, Hearing Research; Target submission date: June 2013.

Hedden, T., \& Gabrieli, J. D. (2004). Insights into the ageing mind: A view from cognitive neuroscience. Nature Reviews Neuroscience, 5(2), 87-96.

Hirsh, IJ. (1952). The Measurement of Hearing. New York, NY: McGraw Hill. 
Holtzer, R., Stern, Y., \& Rakit, B. (2005). Predicting age-related dual-task effects with individual differences on neuropsychological tests. Neuropsychology, 19(1), 1827.

Humes, LE., Dubno, JR., Gordon-Salant, S., Lister, JJ., Cacace, AT., Cruickshanks, KJ., Gates, GA., Wilson, RH., \& Wingfield, A. (2012). Centeral presbycusis: a review and evaluation of the evidence. Journal of the American Academy of Audiology, 23(8), 635-666.

Humes, L.E. (1996). Speech understanding in the elderly. Journal of the American Academy of Audiology, 7, 161-167.

Kiely, K., Gopinath, B., Mitchell, P., Luszez, M., \& Anstey, K. (2012). Cognitive, health, and sociodemographic predictors of longitudinal decline in hearing acuity among older adults. Journal of Gerontology Series A: Biological Sciences and Medical Science, 67(9), 997-1003.

Kirschner, P. (2002). Cognitive load theory: Implications of cognitive load theory on the design of learning. Learning and Instruction, 12, 1-10.

Mattys, S. L., \& Wiget, L. (2011). Effect of cognitive load on speech recognition. Journal of Memory and Language ,65, 145-160.

Mattys, S., Brooks, J., \& Cooke, M. (2009). Recognizing speech under a processing load: Dissociating energetic from informational factors. Cognitive Psychology, 59, 203243.

MedlinePlus. (2012). Age-related hearing loss. U.S. National Library of Medicine, NIH National Institutes of Health. Retrieved January 15, 2013, from http://www.nlm.nih.gov/medlineplus/ency/article/001045.htm

Muller, N.G., \& Knight, R.T. (2006). The functional neuroanatomy of working memory: contributions of human brain lesion studies. Neuroscience, 139, 51-58.

Neisser, U. (1967). Cognitive Psychology. Englewood Cliffs, NJ: Prentice-Hall.

National institute on Deafness and Other Communication Disorders (NIDCD). (1997). Presbycusis. National Institutes of Health, 97-4235. Retrieved January 15, 2013, from https://www.nidcd.nih.gov/health/hearing/pages/presbycusis.aspx

Oldfield, R. C. (1971). The assessment and analysis of handedness: The Edinburgh inventory. Neuropsychologica, 9, 97-113.

Peelle, J.E., Johnsrude, I.S., \& Davis, M.H. (2010). Hierarchical processing for speech in human auditory cortex and beyond. Frontiers in Human Neuroscience, 4(51), $1-3$. 
Pichora-Fuller, M. K., Schneider, B. A.,\& Daneman, M. (1995). How young and old adults listen to and remember speech in noise. Journal of the Acoustical Society of America, 97(1), 593-608.

Pichora-Fuller, M. K.,\& Singh, G. (2006). Effects of age on auditory and cognitive processing: implications for hearing aid fitting and audiologic rehabilitation. Trends in Amplification, 10(1), 29-59.

Pirkl, J. (2009). Characteristics of our aging population. The Demographics of Aging. Retrieved January 15, 2013, from http://www.transgenerational.org/aging/demographics.htm

Rabbit, P., Scott, M., Lunn, M., Thacker, N., Lowe, C., Pendleton, N., et al. (2007). White matter lesions account for all age-related declines in speed not in intelligence. Neuropsychology, 21(3), 363-370.

Raynor, L.A., Pankow, J.S., Miller, M.B., Huang, H., Dalton, D., Klein, R., Klein, B.E.K.,\& Cruickshanks, K.J. (2009). Familial aggregation of age-related hearing loss in an epidemiological study of older adults. American Journal of Audiology, 18(2), 114-118.

Reuter-Lorenz, P.A.,\& Park, D.C. (2010). Human neuroscience and the aging mind: a new look at old problems. Journal of Gerontology: Psychological Sciences, 65B(4), 405-415.

Salthouse, T. (2009). When does age-related cognitive decline begin? Neurobiology of Aging, 30(4), 507-514.

Sarampalis, A., Kalluri, S., Edwards, B.,\& Hafter, E. (2009). Objective measures of listening effort: Effects of background noise and noise reduction. Journal of Speech Language Hearing Research, 52(5), 1230-1240.

Shucknect, H.F. (1964). Further observations on the pathology of presbycusis. Archives of Otolaryngology, 80, 369-382.

Sweller, J., J.J.G., van Merrienboer, and Paas, F.G.W.C. (1998). Cognitive architecture and instructional design. Educational Psychology Review, 10(3), 251-296.

United Nations. (2012). World population ageing: 1950-2050. Department of Economic and Social Affairs, Population Division. Retrieved January 15, 2013, from http://www.un.org/esa/population/publications/worldageing19502050/.

Wiener, J. M. \& Tilly, J. (2002). Population ageing in the United States of America: implications for public programs. International Journal of Epidemiology, 31(4), 776-781. 
Wong, P.C.M, Jin, J.X., Gunasekera, G.M., Abel, R., Lee, E.R., \& Dhar, S. (2009). Aging and cortical mechanisms of speech perception in noise. Neuropsychologia, 47(3), 693-703.

Wong, P.C.M., Ettlinger, M., Sheppard, J.P., Gunasekera, G.M., \& Dhar, S. (2010). Neuroanatomical characteristics and speech perception in noise in older adults. Ear \& Hearing, 31(4), 471-479.

Woodcock, R. W., Mather, N., \& McGrew, K. S. (2001). Woodcock-Johnson III Tests of Cognitive Abilities Examiner's Manual. Itasca, IL : Riverside.

Young, G., Zavelina, L., \& Hooper, V. (2008). Assessment of workload using NASA task load index in perianesthesia nursing. Journal of Perianesthesia Nursing, 23(2), 102-110.

Yueh, B., Shapiro, N., MacLean, C.H., \& Shekelle, P.G. (2003). Screening and management of adult hearing loss in primary care. Journal of the American Medical Association, 289(15), 1976-1985. 


\section{APPENDIX A. PARTICIPANT INSTRUCTIONS}

\section{Condition A}

For this task, you will hear sentences in noise and you will see instructions on screen that will prompt you to search for a letter. Repeat the last word in the sentence, after hearing each sentence, and each time the letter is on the screen click the green button in front of you.

\section{Condition B}

For this task, you will hear sets of words in noise and you will see instructions on screen that will prompt you to search for a letter. Once a set of words has been presented, you will hear the word "START". When you hear the word "START" the set is over, and you must recall each word in the set, and each time the letter is on the screen click the green button in front of you.

- So, here is an example

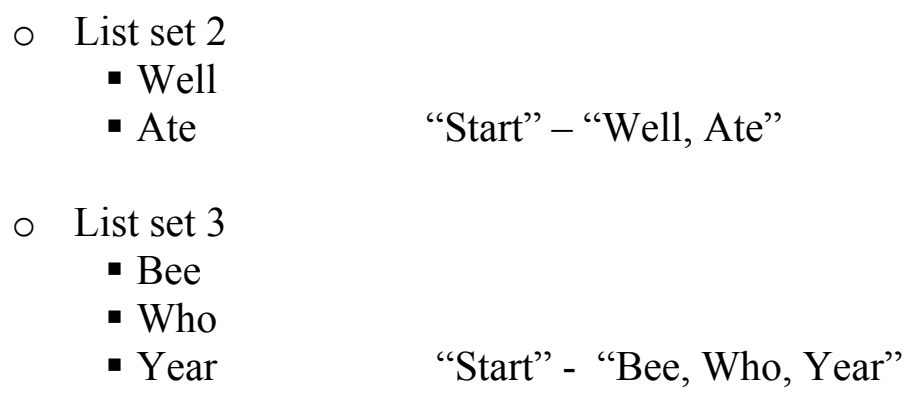

- If it's possible, you say the last words in the order in which they were presented. If you can't remember them in order, you can say them in any order, but you should try not to start with the last word first, unless it is the only one you can remember.

○ Your goal is to say back as many of the last words in the set as possible.

- The set size will change throughout the task. Do not start repeating the words until you hear the word "START".

\section{Condition C}

During this task, you will hear sets of sentences in noise and you will see instructions on screen that will prompt you to search for a letter. Once a set of sentences has been presented, you will hear the word "START". When you hear the word "START" the set is over, and you must recall the last word of each of the sentences in the set.

- So, here is an example

- List set 2

- The sky is blue.

- The grass is green. "Start" - "Blue, Green" 
$\circ \quad$ List set 3

- The door is broken.

- The banana is old.

- The yellow car is new. "Start" - "Broken, Old, New"

- If it's possible, you say the last words in the order in which they were presented. If you can't remember them in order, you can say them in any order, but you should try not to start with the last word first, unless it is the only one you can remember.

o Your goal is to say back as many of the last words in the set as possible.

- The set size will change throughout the task. Do not start repeating the words until you hear the word "START".

Instructions for Conditions $B$ and $C$ were adapted from the work of Daneman and Carpenter (1980). 


\section{APPENDIX B. GROUP DATA ACROSS DUAL TASK CONDITIONS}

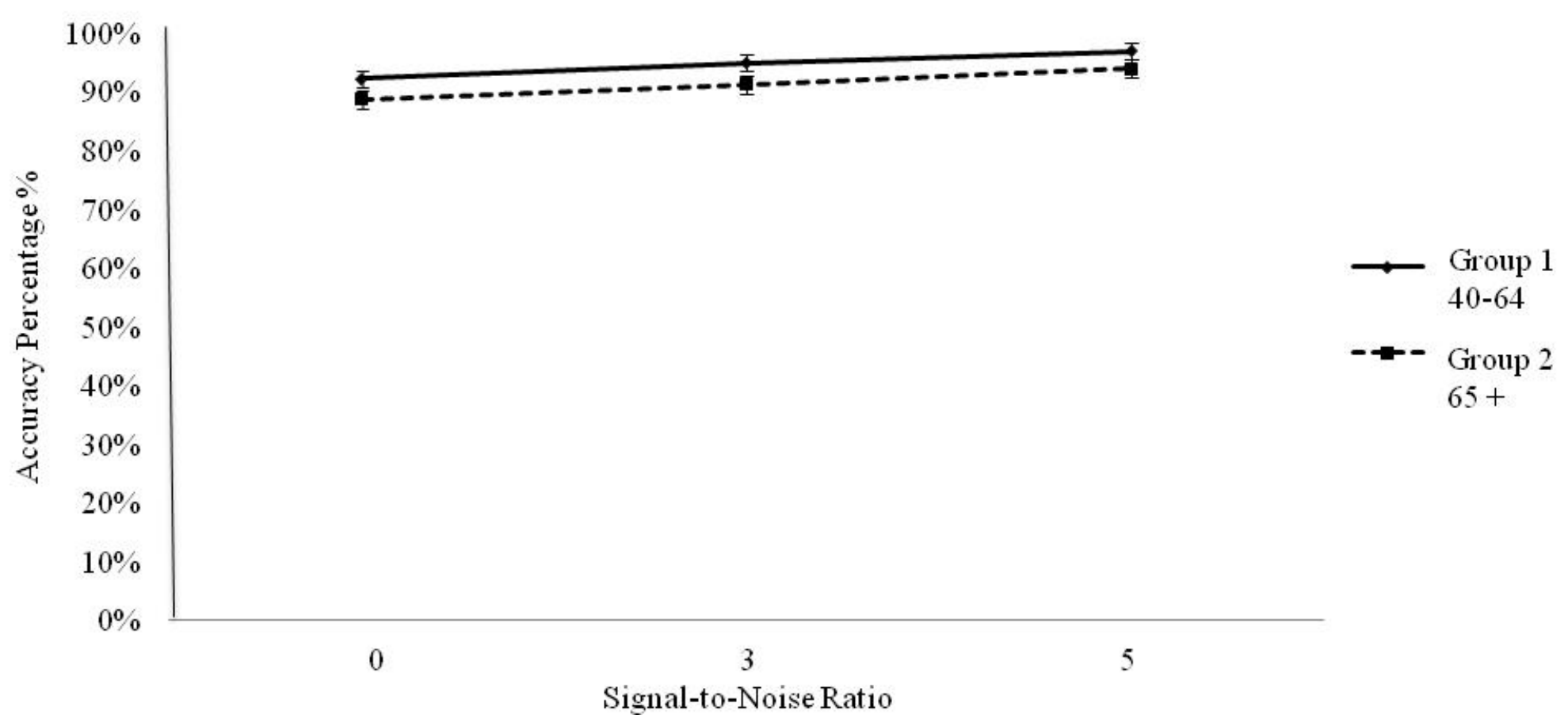

Figure B-1. Group 1 (Younger) and Group 2 (Older) Auditory Accuracy during Simple Dual Task 


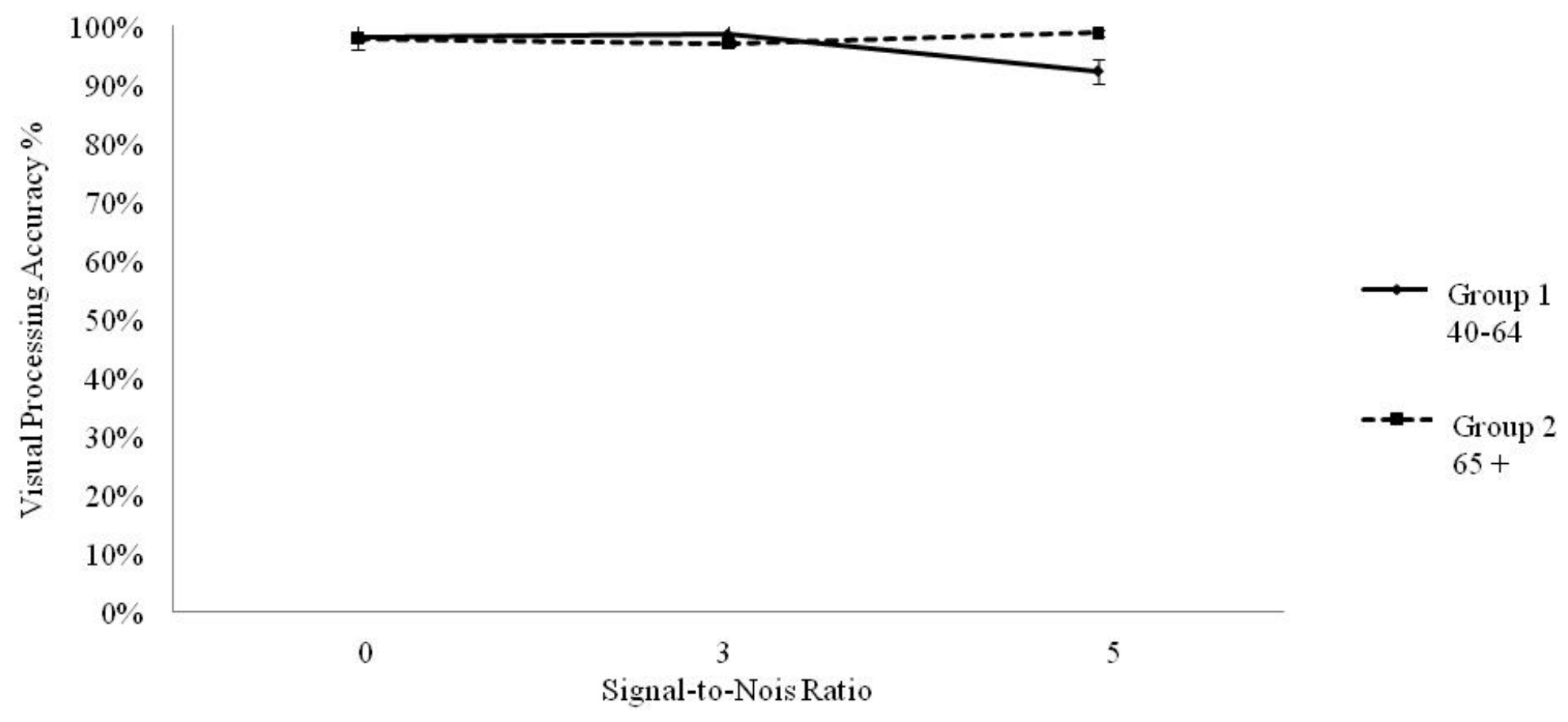

Figure B-2. Group 1 (Younger) and Group 2 (Older) Visual Processessing Accuracy during Simple Dual Task 


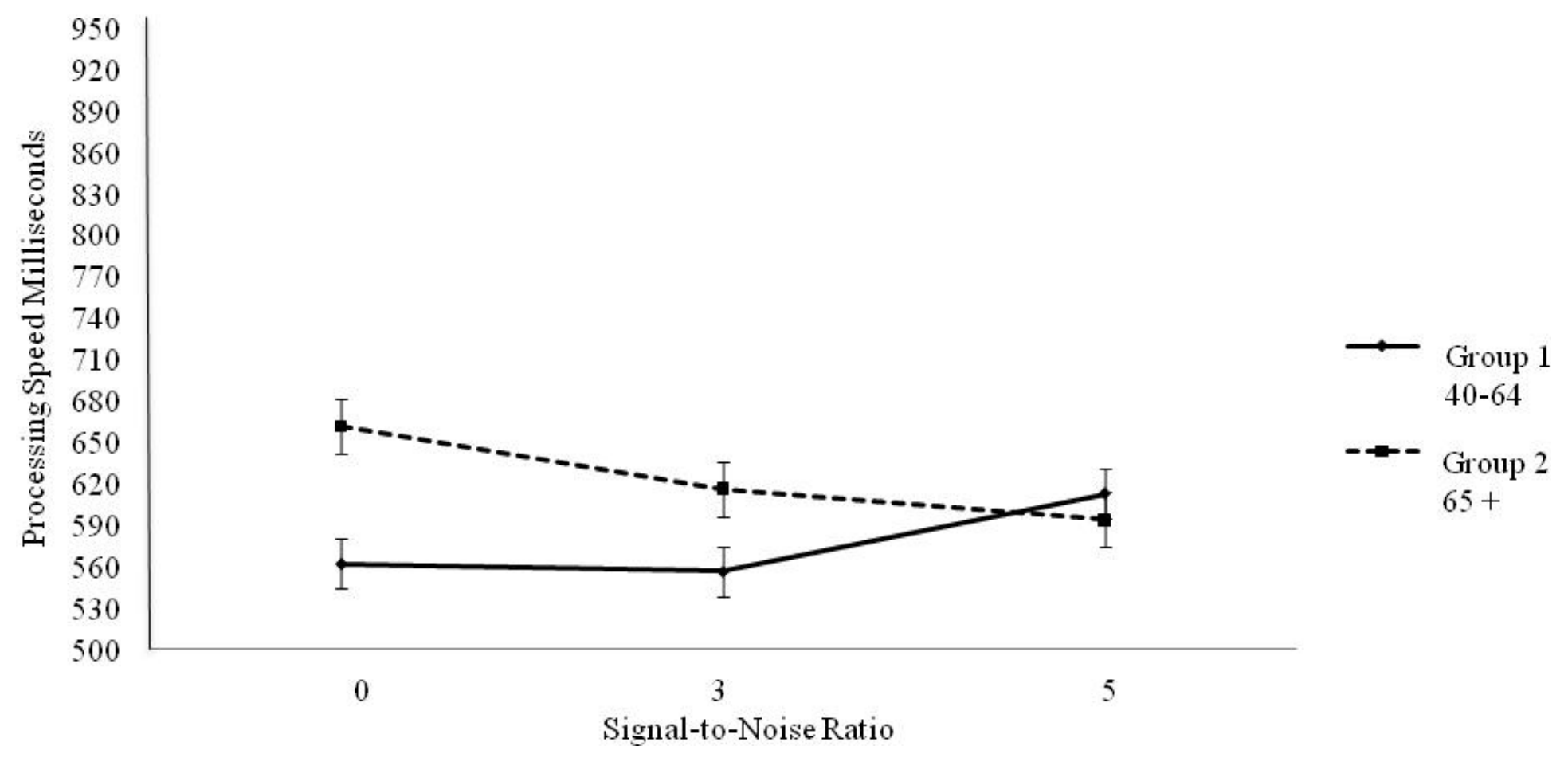

Figure B-3. Group 1 (Younger) and Group 2 (Older) Visual Reaction Time during Simple Dual Task 


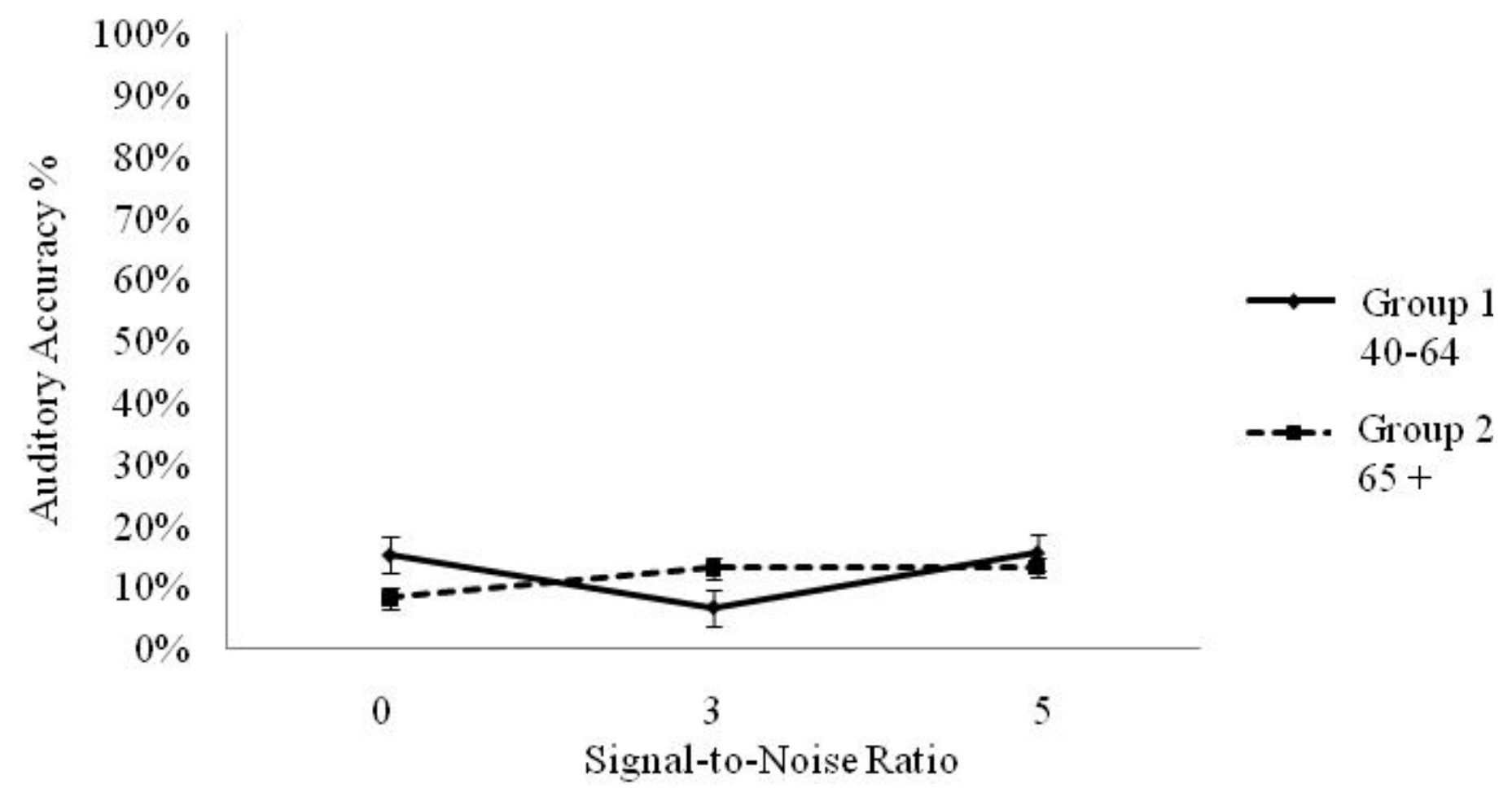

Figure B-4. Group 1 (Younger) and Group 2 (Older) Auditory Accuracy during Moderate Dual Task 


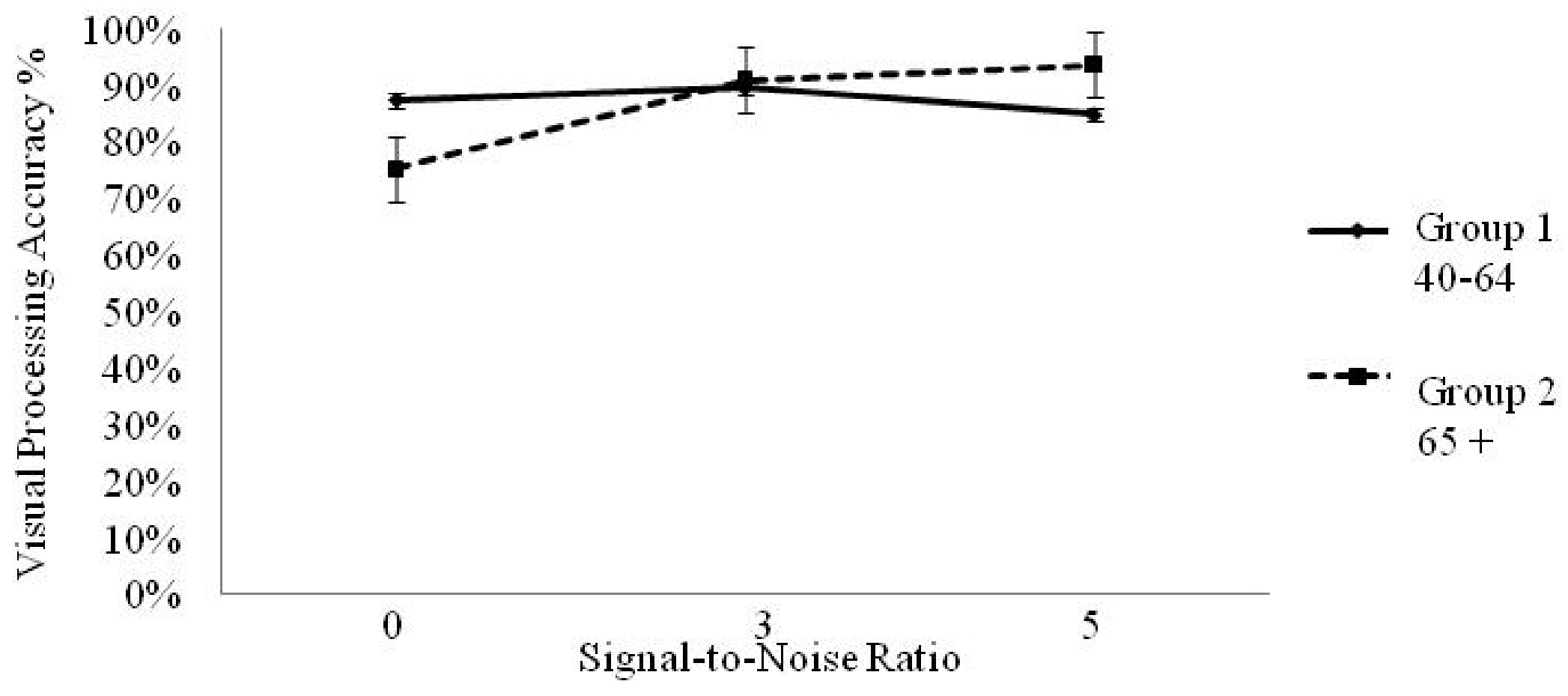

Figure B-5. Group 1 (Younger) and Group 2 (Older) Visual Processing Accuracy during Moderate Dual Task 


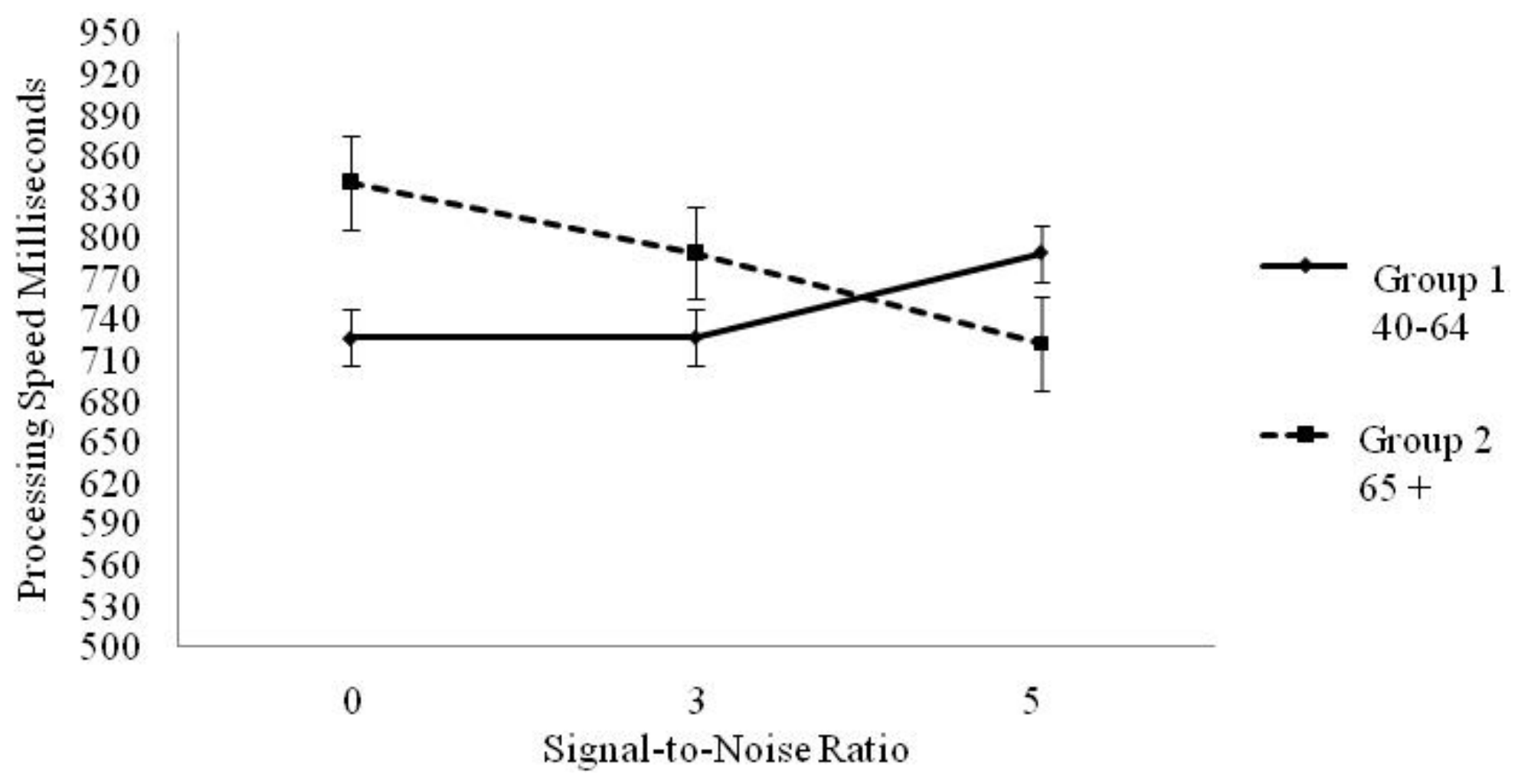

Figure B-6. Group 1 (Younger) and Group 2 (Older) Visual Reaction Time during Moderate Dual Task 


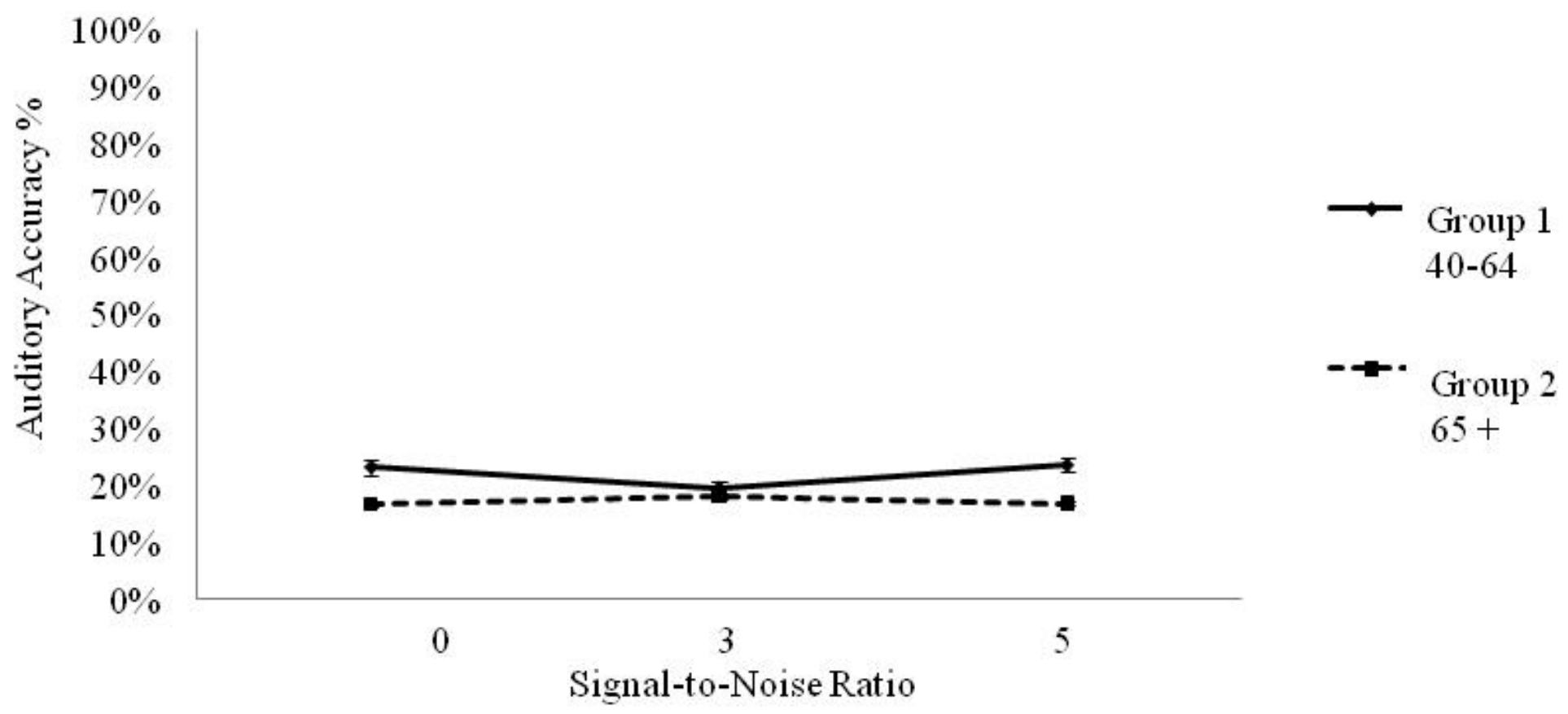

Figure B-7. Group 1 (Younger) and Group 2 (Older) Auditory Accuracy during Complex Dual Task 


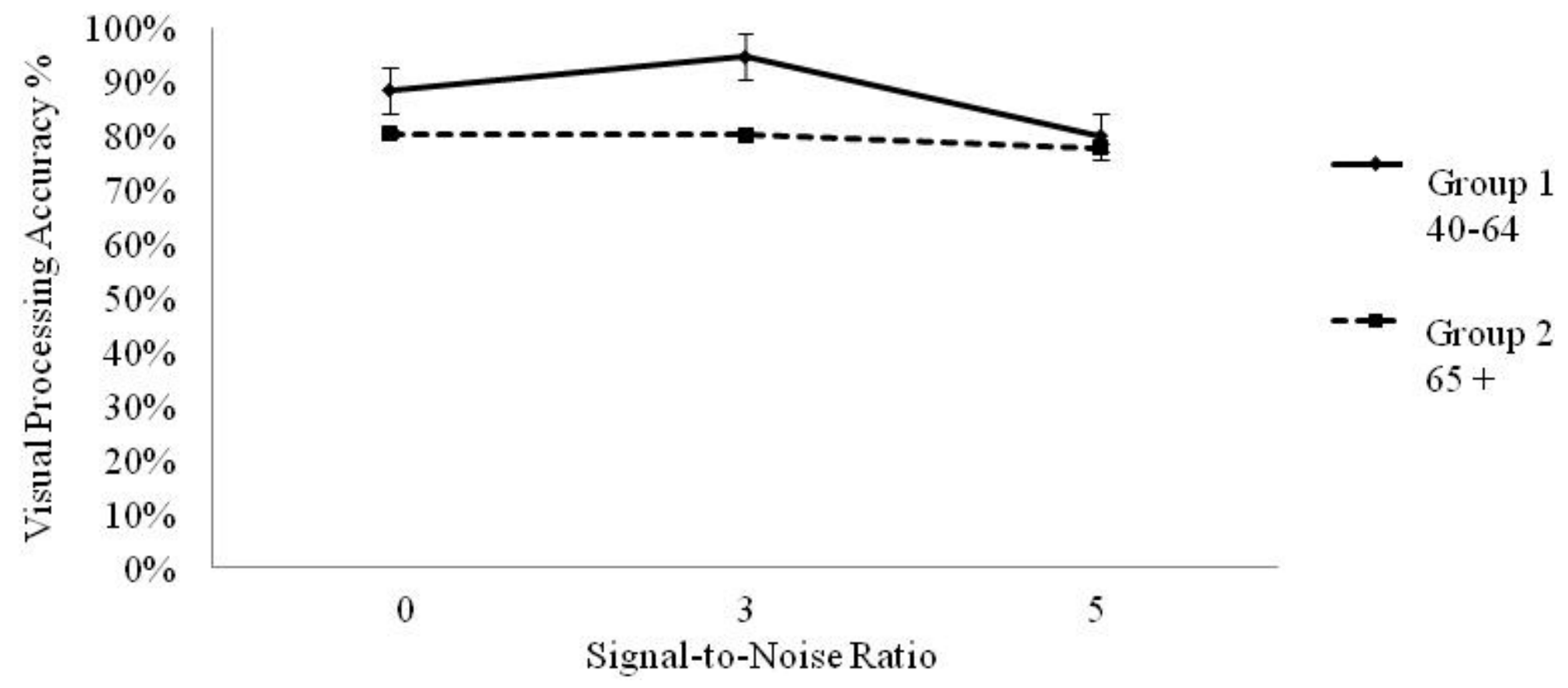

Figure B-8. Group 1 (Younger) and Group 2 (Older) Visual Processing Accuracy during Complex Dual Task 


\section{Complex DT Processing Speed}

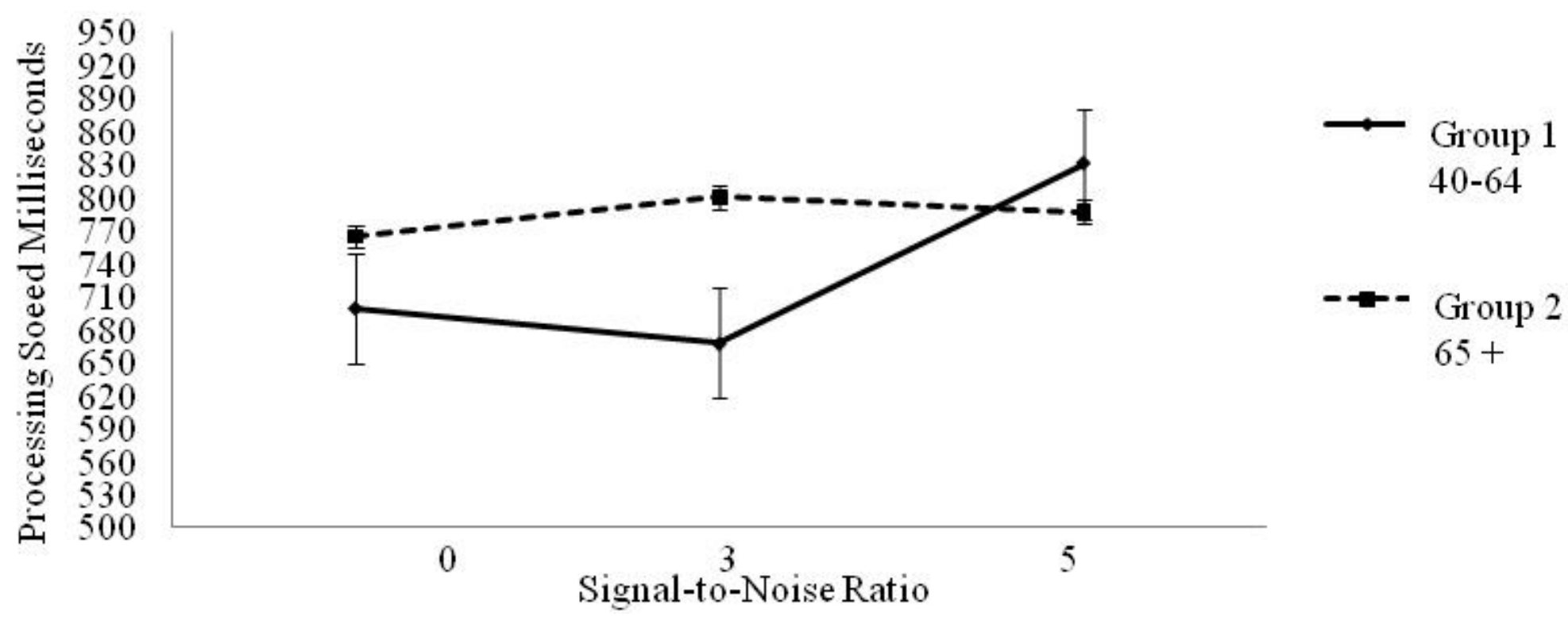

Figure B-9. Group 1 (Younger) and Group 2 (Older) Visual Reaction Time during Complex Dual Task 


\section{VITA}

Jennine Harvey was born in 1982 in Tacoma, Washington. She graduated from Lincoln Park Academy in 2001, and received her Bachelor's and Master's Degrees from the University of Central Florida in 2004 and 2006. Jennine will receive her Ph.D. from the University of Tennessee Health Science Center Speech and Hearing Science Program in May 2013. Jennine Harvey is an assistant professor at Illinois State University in the Department of Communication Sciences \& Disorders, and is a certified speech-language pathologist, specializing in adult neurogenic communication disorders . She conducts research in the area of cognition, speech in noise, aphasia, and aging. 